\title{
1 TITLE: Proprioceptive and visual feedback responses in macaques exploit goal redundancy
}

2

\section{3}

4

5

6

\section{Authors and affiliations}

Kevin P. Cross ${ }^{1,2 *}$, Hui Guang ${ }^{2}$, Stephen H. Scott ${ }^{2,3,4}$.

${ }^{1}$ Neuroscience Center, University of North Carolina, Chapel Hill, North Carolina 27599, ${ }^{2}$ Centre for Neuroscience Studies, ${ }^{3}$ Department of Biomedical and Molecular Sciences and ${ }^{4}$ Department of Medicine, Queen's University, Kingston, ON, K7L 3N6, Canada.

*Corresponding author: 13kc18@queensu.ca

\section{Acknowledgements}

We thank Kim Moore, Simone Appaqaq, Catherine Crandell, Jordan Miller, Ethan Heming, and Helen Bretzke for their laboratory and technical assistance. This work was supported by grants from the Canadian Institute of Health Research to SHS. KPC was supported by an Ontario Graduate Scholarship. SHS was supported by a GSK chair in Neuroscience.

\section{Declaration of Interests}

SHS is co-founder and CSO of Kinarm which commercializes the robotic technology used in the present study. 


\section{$32 \quad$ Abstract}

A common problem in motor control concerns how to generate patterns of muscle activity when

there are redundant solutions to attain a behavioural goal. Optimal feedback control is a theory that has

guided many behavioural studies exploring how the motor system incorporates task redundancy. This

theory predicts that kinematic errors that deviate the limb should not be corrected if one can still attain

the behavioural goal. Several studies in humans demonstrate that the motor system can flexibly

integrate visual and proprioceptive feedback of the limb with goal redundancy within $90 \mathrm{~ms}$ and $70 \mathrm{~ms}$,

respectively. Here we show monkeys (Macaca mulatta) demonstrate similar abilities to exploit goal

redundancy. We trained four male monkeys to reach for a goal that was either a narrow square or a

wide, spatially redundant rectangle. Monkeys exhibited greater trial-by-trial variability when reaching

to the wide goal consistent with exploiting goal redundancy. On random trials we jumped the visual

43 feedback of the hand and found monkeys corrected for the jump when reaching to the narrow goal and

44 largely ignored the jump when reaching for the wide goal. In a separate set of experiments, we applied

mechanical loads to the monkey's arm and found similar corrective responses based on goal shape.

Muscle activity reflecting these different corrective responses were detected for the visual and

mechanical perturbations starting at $\sim 90$ and $\sim 70 \mathrm{~ms}$, respectively. Thus, rapid motor responses in

of goal-directed motor action and motor redundancy. 
$\underline{\text { Introduction }}$

A common problem in motor control concerns how to select muscle commands when there are many different ways to attain a behavioural goal (Bernstein, 1967; Flash and Hogan, 1985; Sporns and Edelman, 1993; Scholz and Schöner, 1999; Scholz et al., 2000; Latash, 2012). For example, successfully reaching for an object can involve many different trajectories of the limb to the goal, and thus, many different patterns of muscle activity. Optimal feedback control (OFC) provides a framework for how to select motor commands among a family of redundant solutions (Todorov and Jordan, 2002; Scott, 2004). OFC selects motor commands to optimize a cost function that balances successfully completing the behavioural goal with the cost of movement (e.g. energy, noise). Importantly, these controllers abide by the minimum intervention principle where kinematic errors that arise during movement are only corrected if they interfere with the behavioural goal. Or alternatively stated, errors that deviate the plant along redundant trajectories should not be corrected. As a result, variability accumulates along redundant task dimensions.

Several studies demonstrate that the motor system exploits task redundancies similar to OFC controllers (Diedrichsen, 2007; Dimitriou et al., 2012; Cluff and Scott, 2015; Weiler et al., 2015, 2016). A common approach is to have participants reach to a spatially redundant goal such as a wide rectangular bar (Knill et al., 2011; Nashed et al., 2012; de Brouwer et al., 2017; Keyser et al., 2017, 2019). Participants exhibit greater trial-to-trial variability in their reach endpoints when reaching for a wide as compared to a narrow goal. The increased variability also exhibits structure as variability primarily accumulates along the wide (redundant) axis of the goal. Furthermore, displacements to the visual feedback of the hand (cursor jump) are fully corrected by participants when reaching for a narrow goal and are corrected less when reaching for the wide goal if the displacement is along the redundant axis (Knill et al., 2011; de Brouwer et al., 2017; Cross et al., 2019). Differences in these corrective responses arise in muscle activity 90ms after the jump (Franklin and Wolpert, 2008; Cross 
et al., 2019). Similar corrective responses occur when mechanical loads are applied to the limb with differences between corrective responses starting $~ 70 \mathrm{~ms}$ after the load (Nashed et al., 2012; Lowrey et al., 2017; Keyser et al., 2019). However, despite the prevalence of OFC as a theory of motor control, we know little about the neural circuits used to generate OFC-like behaviours.

behavioural tasks must be translated into animal models that allow for invasive neural recordings such as rhesus monkeys or rodents. To perform most behavioural tasks the behaviour of the animal must be shaped using reward over the course of tens of thousands of trials. This excessive training along with behavioural shaping results in behaviour that is highly reproducible on a trial-by-trial basis which provides advantages when analyzing noisy neural activity. However, it is unclear if this highly reproducible behaviour comes at the cost of behavioural flexibility. For example, Bizzi and colleagues trained monkeys to reach to a goal and applied an assistive mechanical load that pushed the monkey's limb towards the goal (Bizzi et al., 1982, 1984). Monkeys corrected by actively resisting the load in what appeared to be an attempt to return the limb to the original trajectory to the goal. In contrast, OFC models and humans performing a similar task do not return to the original trajectory and instead allow the load to push the hand towards the goal thereby exploiting the fact that there are redundant trajectories (Cluff and Scott, 2015). Thus, humans can exploit task redundancies, however it is unclear whether monkeys are capable of exploiting redundancies in an experimental setting due to overtraining and behavioural shaping.

Here, we investigated whether monkeys could learn to exploit the spatial redundancy of a goal

94 during reaching. We found monkeys exhibited greater variability in their reach endpoints on

95 unperturbed trials. Further, monkeys corrected less for visual and mechanical perturbations of the limb when reaching for the spatially redundant target, consistent with exploiting goal redundancy. Muscle 
bioRxiv preprint doi: https://doi.org/10.1101/2022.02.11.480080; this version posted February 14,2022 . The copyright holder for this preprint (which was not certified by peer review) is the author/funder, who has granted bioRxiv a license to display the preprint in perpetuity. It is made available under aCC-BY-NC-ND 4.0 International license.

97 recordings indicated that feedback responses to visual and mechanical perturbations reflected goal

98 redundancy within $<100 \mathrm{~ms}$. 
$\underline{\text { Methods }}$

100

101

102

103

104

105

106

107

108

109

110

111

112

113

114

115

Four male monkeys (Macaca mulatta, 10-20kgs) were trained to sit in a primate chair and place their upper arm into a robotic exoskeleton (Scott, 1999, Kinarm, Kingston, Canada). The robot constrained the monkey's arm to move in a two-dimensional plane and included a virtual reality system that could display virtual targets and visual feedback of the limb. Experiments were approved by the Queen's University Research Ethics Board and Animal Care Committee.

Behavioural task for visual perturbations. Monkeys were trained to make goal-directed reaches to targets in the virtual environment. Visual feedback of the hand was provided by a white cursor (radius $=0.8 \mathrm{~cm}$ ) aligned with the monkey's index fingertip. At the start of each trial, a start target (square, side length $=1.2 \mathrm{~cm}$ ) appeared and the monkey was required to reach and hold their hand at the target for $750-1500 \mathrm{~ms}$. Next, a goal target appeared that was located $8 \mathrm{~cm}$ lateral, and $4.4 \mathrm{~cm}$ in front of the start goal (total reach distance $9.2 \mathrm{~cm}$, Figure 1A). To reach the goal from the starting position monkeys had to primarily extend their elbow. When the monkey's hand left the start target, they had $900 \mathrm{~ms}$ to reach and stabilize their hand within the goal for 500ms. The goal could either be a narrow rectangle (length $2 \mathrm{~cm}$, width $2.2 \mathrm{~cm}$ ) or a wide rectangle with its long axis oriented perpendicular to the reach axis (straight path between start and middle of goal target; wide goal dimensions length $12 \mathrm{~cm}$ width $2.2 \mathrm{~cm}$ ). On random trials, the cursor jumped orthogonal to the reach axis (orthogonal reach axis) once the hand was $2 \mathrm{~cm}$ from the start target and on these trials monkeys were given an additional $500 \mathrm{~ms}$ to reach to the goal. For monkeys $\mathrm{M}$ and $\mathrm{A}$, the cursor jumped $\pm 4 \mathrm{~cm}$ and for monkeys $\mathrm{T}$ and $\mathrm{C}$ the cursor jumped $\pm 3.5 \mathrm{~cm}$. Within a block of trials there were 6 no-jump reaches $(3$ for both goal shapes), and 4 cursor-jump trials (2 directions x 2 goal shapes). Monkeys completed 10-25 blocks in a given recording session. 
Behavioural task for mechanical perturbations. The task was similar to the cursor perturbation task, but

124 there were several changes to the shape and size of the goal targets. First, the size of the redundant axis

125 of the wide goal was $28 \mathrm{~cm}$ long (Figure 1B) and it was shaped like an arrowhead composed of two

126 rectangles overlapping at the edges and at an angle of $110^{\circ}$ with respect to each other. We chose this

127 configuration as it brought the edges of the goal closer in proximity to the monkey's arm, thus making

128 it easier to reach on perturbation trials. On random trials mechanical loads were applied to the joints to

129 displace the limb approximately orthogonal to the reach axis (Monkey $\mathrm{M}|\mathrm{A}| \mathrm{T} \mid \mathrm{C}$ pushes limb away from

130 body: shoulder $0.5|0.45| 0.4 \mid 0.3 \mathrm{Nm}$ elbow $0|0| 0 \mid 0 \mathrm{Nm}$; pushes limb towards the body: shoulder $-0.5 \mid-$

$1310.45|-0.4|-0.3 \mathrm{Nm}$ elbow $0.15|0.1| 0.07 \mid 0.14 \mathrm{Nm}$; + flexion loads, - extension loads). Due to the influence

132 of limb mechanics (i.e. limb inertia, limb geometry), loads were adjusted for each monkey to generate

133 roughly similar hand deviations. Cursor feedback was temporarily removed for 200ms when the load

134 was applied. We also increased the number of unperturbed trials to reduce anticipatory corrections to

135 the mechanical loads. Within a block of trials there were 8 no-load reaches ( 4 for both goal shapes), and

1364 load trials (2 directions x 2 goal shapes). Monkeys completed 10-15 blocks in a given recording

137 session.

139 Estimating visual onsets. On cursor-jump trials, there is approximately a 20-49ms delay between when

140 the command was sent to jump the cursor and when it actually updated the screen. We estimated this

141 delay on a trial-by-trial basis by using photodiodes placed at the side of the screen and flashing white

142 squares coincident to the photodiode locations when the cursor jumped.

144 Muscle recordings. We implanted Monkey M with a 32-channel chronic EMG system (Link-32, Ripple

145 Neuro, Salt Lake City UT). The system had 8 leads that were inserted into the muscle belly and were

146 attached to a processor. Each lead had 4 separate contacts for recording intramuscular activity 
147 (impedance $20 \mathrm{kOhms}$ ). The processor was implanted under the skin and located near the midline of

148 the back at the mid-thoracic level. Muscles implanted were brachioradialis, brachialis, the lateral and

149 long heads of the triceps, biceps (long head), pectoralis major, and anterior and posterior deltoids. An

150 external receiver was secured to the skin over the processor using magnets in the receiver and

151 processor. The external receiver was capable of powering the internal processor and EMG signals were

152 transmitted through the skin from the processor to the receiver by photodiodes. The signals were then

153 relayed to the Grapevine Neural Interface Processor (Ripple Neuro, Salt Lake City, UT), bandpass

154 filtered $(15-375 \mathrm{~Hz})$ and recorded at $2 \mathrm{kHz}$.

155 In Monkey $\mathrm{C}$ we recorded muscle activity using surface EMG electrodes (Delsys, Natwick MA,

156 USA). We recorded activity from brachalis, biceps, and the lateral and long head of the triceps. Activity

157 was sampled at $1 \mathrm{kHz}$, bandpass filtered at $20-200 \mathrm{~Hz}$ using a $6^{\text {th }}$ order Butterworth filter and rectified.

159 Kinematic recordings. The shoulder and elbow angles, angular velocities and angular accelerations

160 were recorded by either a 128-Channel Neural Signal Processor (Blackrock Microsystems, Salt Lake

161 City, UT) at $1 \mathrm{kHz}$, or by the Grapevine Neural Interface Processor at $30 \mathrm{kHz}$.

162

$163 \quad$ Data and statistical analyses

164 Kinematic analysis. The endpoint position of the reach was calculated by finding the hand position at

165 the end of the trial (timepoint after $500 \mathrm{~ms}$ hold period). This hand position was then projected onto the

166 redundant axis of the wide goal by finding the location on the redundant axis that had the shortest

167 distance to the hand position. The endpoint position was also made relative to the center of the relative

168 target as there were small positional offsets between the narrow and wide targets.

169 We estimated the timing of the kinematic corrections using the hand velocity along the

170 orthogonal reach axes, which was defined as the velocity component perpendicular to the straight path 
connecting the start and middle of the goal (reach axis, Figure 1). The hand velocity was aligned to the

172 onset of the perturbation (cursor jump or mechanical load) or the equivalent time point on no-

173 perturbation trials (faux jump or faux load). The average velocity on no-perturbation trials was

174 subtracted from the velocity on perturbation trials resulting in the change $(\Delta)$ in hand velocity. Timing

175 of when the hand velocity differentiated based on goal shape was determined using receiver-operator

176 characteristics (ROC) (Corneil et al., 2004; Gu et al., 2016; Pruszynski et al., 2016; Cross et al., 2019).

177 At each time point we generated an ROC curve between the hand velocities for the narrow and wide

178 goals. The area under the ROC curve reflects how discriminable the trials for the narrow and wide

179 goals are and can range from 0.5 , indicating chance discrimination, to 0 and 1 indicating perfect

180 discrimination. We found the first time point that had an area $>0.8 /<0.2$ and that was maintained above

181 this threshold for 10 consecutive time points (10ms). We then traced backwards in time to the first time

182 point that fell below/above $0.6 / 0.4$ (knee).

183

184 EMG analysis. For indwelling muscle activity recorded from Monkey $\mathrm{M}$ we down sampled activity to

$1851 \mathrm{kHz}$. For each lead, we computed differential signals from the two most proximal contacts and two

186 most distal contacts relative to the processor. For muscle activity recorded using surface electrodes

187 from Monkey C, we only included recordings where perturbation-related activity was detected.

188 The differential signals were rectified and low-passed filtered with a $6^{\text {th }}$ order zero-phase lag

189 Butterworth filter at 50Hz. Muscle activities were aligned to the onset of the perturbation. Muscle

190 activities were then trial-averaged and the activities on no-perturbation trials were subtracted from

191 perturbation trials to yield the change in activity caused by the perturbation. Muscle activities were

192 normalized by the mean perturbation-related activity from 0-300ms after perturbation onset. 
194 Analysis of no-perturbation trials. We compared magnitudes of muscle activity between reaches for

195 the narrow and wide goals on no-perturbation trials. Activities were averaged in the epoch starting

196 200ms before the faux-jump/faux-load onset until 200ms after the onset (movement epoch). A two-

197 sample t-test identified muscles that were significantly different between the narrow and wide goals

198 ( $\mathrm{p}<0.01)$. We also examined how temporally correlated muscle activities were between the narrow and

199 wide goals during the movement epoch using Pearson's correlation coefficient. We compared the

200 observed distribution across muscles with a shuffled distribution where correlations were computed

201 between randomly selected muscles.

202

Preferred direction and perturbation-sensitive criteria. A muscle's preferred perturbation direction was

calculated by averaging the perturbation-related activities over the first $300 \mathrm{~ms}$ after the perturbation

onset for reaches to the narrow goal. The direction with the largest activity over this epoch was defined

as the preferred direction. The same preferred direction was used for both the narrow and wide goals.

Perturbation-sensitive muscle samples were identified using a two-sample t-test comparing the

activity on unperturbed trials with activity on perturbation trials in the muscle's preferred direction in

the epoch of $0-300 \mathrm{~ms}$ after the perturbation onset. This was applied twice for each muscle, one test for

each target shape. Muscles were classified as "perturbation sensitive" if $\mathrm{p}<0.05$ (Bonferroni correction

211 factor 2).

213 Epoch analysis. We compared how perturbation-related activities differed between goal shapes over

214 time. For the mechanical perturbations we divided each muscle's activity into epochs of 20-50ms, 50-

$21575 \mathrm{~ms}, 75-100 \mathrm{~ms}$ and 120-180ms based on previous work (Lee and Tatton, 1975; Crago et al., 1976;

216 Bonnet, 1983; Omrani et al., 2014; Pruszynski et al., 2014). For the cursor perturbations we considered 217 the same epochs but shifted 50ms forward in time (70-100ms, 100-125ms, 125-150ms and 170-230ms) 
218 to account for the fact that muscle activities respond to visual feedback $\sim 50 \mathrm{~ms}$ slower than for

219 proprioceptive feedback (Franklin and Wolpert, 2008; Pruszynski et al., 2010, 2016; Dimitriou et al.,

220 2013; Cross et al., 2019). For each muscle, we applied a two-way ANOVA with time (4 levels) and goal

221 shape (2 levels) as factors. Muscles had significant goal-shape modulation if there was a significant

222 main effect for goal shape or if there was an interaction effect ( $\mathrm{p}<0.05$ Bonferroni correction 2$)$.

223 Significant interaction effects were decomposed using post-hoc t-tests by comparing across goal shape

224 within each time epoch ( $\mathrm{p}<0.05$ Bonferroni correction 4).

226 Population signal. We calculated the population signal by averaging the activities for all perturbation227 sensitive muscle samples in their preferred directions. A difference signal was calculated by 228 subtracting the activities for the wide goal from the narrow goal for all perturbation-sensitive muscle 229 samples followed by averaging across samples. The onset for the population signals were estimated by 230 calculating the mean and standard deviation (SD) of the baseline activity (300ms before perturbation 231 onset) and finding the first time point that exceeded the mean by 3SD for 20 consecutive time points 232 (Omrani et al., 2016). 
$\underline{\text { Results }}$

Our goal was to develop a reaching task to examine if monkeys could exploit the spatial

redundancy of a goal. The original tasks performed by humans involved a multi-joint reach directly in

translate this task into the monkey was not successful as the monkey exhibited substantial bias towards

reaching one end of the bar (note: humans can also show a similar bias, albeit smaller, see Keyser et al.,

2017). Instead, we focused on reaches that primarily required an elbow extension movement, which

produced more consistent behaviour (Figure 1). Below, we first describe the experiments that involved

cursor perturbations followed by the experiments that involved mechanical perturbations.

\section{Experiment 1: Goal redundancy and feedback responses to cursor jumps}

We trained four monkeys to reach to a goal that could be either a narrow square (Figure 1A top)

or a wide rectangle (Figure 1A bottom; trials interleaved). For monkeys $\mathrm{M}|\mathrm{A}| \mathrm{T} \mid \mathrm{C}$, we recorded

$6|7| 14 \mid 12$ behavioural sessions of the animals performing the task on separate days which were spread

across 1-2 years for each monkey and monkeys were able to perform the task with high efficiency

(success rates monkeys M|A|T|C: narrow targets=95|98|89|97\%, wide targets=96|100|92|98\%).

Figure 2A shows the hand paths for monkey $M$ to the narrow (left) and wide (right) goals from one recording session. There was greater trial-by-trial variability in the hand position during the reach

resulted in the reach endpoints exhibiting greater dispersion along the redundant axis of the goal for reaches to the wide goal compared with reaches to the narrow goal (Figure 2A-C). Across recording sessions, there was no significant difference in mean endpoint position for monkeys $\mathrm{M}$ and $\mathrm{C}$, whereas there was a $0.7 \mathrm{~cm}$ and $1.3 \mathrm{~cm}$ difference between endpoint positions for the narrow and wide goals for monkeys A $(t(6)=3.8, p=0.01$; Figure $2 \mathrm{D})$ and $\mathrm{T}(\mathrm{t}(13)=9.5, \mathrm{p}<0.001)$, respectively. In contrast, the 
257 the narrow goal for monkeys M, A, T, and C, respectively (Figure 2E; paired t-test Monkey M|A|T|C

$\mathrm{t}(5)=8.3|\mathrm{t}(6)=4.6| \mathrm{t}(13)=6.8|\mathrm{t}(11)=10.9, \mathrm{p}<0.001| \mathrm{p}=0.004|\mathrm{p}<0.001| \mathrm{p}<0.001)$

Next, we examined the unperturbed hand velocities for reaches to the narrow and wide goals.

260 Figures $3 \mathrm{~A}$ and B (inset) show the unperturbed hand velocities during the same recording session along 261 the reach and the orthogonal reach (ortho. reach) axes, respectively. There was a small increase in the 262 peak hand velocity for reaches to the wide goal as compared to reaches to the narrow goal. Across 263 recording sessions, we found a small increase in hand speed for the wide goal for monkey M $264($ narrow $=0.48 \mathrm{~m} / \mathrm{s}$, wide $=0.51 \mathrm{~m} / \mathrm{s}$, paired $\mathrm{t}$-test $\mathrm{t}(5)=3.6, \mathrm{p}=0.02)$, a small decrease for the wide goal for 265 monkey $\mathrm{T}$ (narrow $=0.35 \mathrm{~m} / \mathrm{s}$, wide $=0.32 \mathrm{~m} / \mathrm{s}, \mathrm{t}(13)=2.5, \mathrm{p}=0.02)$, and no effect of goal shape on hand 266 speed for monkeys A and C (Monkey A|C: narrow $=0.52 \mid 0.49 \mathrm{~m} / \mathrm{s}$ and wide $=0.52 \mid 0.5 \mathrm{~m} / \mathrm{s}$, $\mathrm{t}(6)=2.0|\mathrm{t}(11)=0.2, \mathrm{p}=0.1| \mathrm{p}=0.8)$.

Next, we examined how goal redundancy affected corrective responses for unexpected cursor jumps. Figure 4A shows monkey M's hand paths on cursor-jump trials for the narrow and wide goals. There is a clear correction for the cursor jump when reaching for the narrow goal and little if any correction when reaching for the wide goal. There was also greater trial-by-trial variability in the hand position during the reach to the wide goal (Figure 4B), indicating that the monkey did not simply learn to reach for a particular location on the goal during cursor-jump trials. We calculated the differences between reach endpoints on cursor-jump trials and the mean of the unperturbed reach endpoint (change in reach endpoint, Figure 4C). For the narrow goal, the change in reach endpoints for either cursor jump direction (blue solid line: jumps away from body; blue dashed line: jumps towards body) were narrow distributions centered near zero (mean: solid $0.26 \mathrm{~cm}$, dashed $-0.55 \mathrm{~cm}$ ) indicating that monkeys ended their reach at almost the same location as on unperturbed trials. In contrast, for the wide goal the change in reach endpoints generated wide distributions that were centered $\sim 3 \mathrm{~cm}$ from the zero-mark 280 (red solid $2.9 \mathrm{~cm}$, red dashed $-3.6 \mathrm{~cm}$ ) indicating that the monkey largely ignored the cursor jump. 
281 Across sessions and in both jump directions, there was a greater change in endpoint position for the 282 wide goal than the narrow goal (Figure 4D, F; paired t-tests: jumps away from body | towards body 283 monkey $\mathrm{M} \mathrm{t}(5)=7.6 \mid \mathrm{t}(5)=11$, monkey $\mathrm{A} \mathrm{t}(6)=49 \mid \mathrm{t}(6)=48.9$, monkey $\mathrm{T} \mathrm{t}(13)=33 \mid \mathrm{t}(13)=19$, monkey C $284 \mathrm{t}(11)=24, \mid \mathrm{t}(11)=23.8, \mathrm{p}<0.001$ for all comparisons). Furthermore, the standard deviation in reach 285 endpoints was also significantly greater for reaches to the wide goal for monkey M (Figure 4E, G; 286 jumps away from body|towards body: paired t-test: $\mathrm{t}(5)=7.9|\mathrm{t}(5)=8.4, \mathrm{p}<0.001| \mathrm{p}<0.001)$, monkey $\mathrm{T}$ $287(\mathrm{t}(13)=6.4|\mathrm{t}(13)=5.6, \mathrm{p}<0.001| \mathrm{p}<0.001)$ and monkey $\mathrm{C}(\mathrm{t}(11)=8.4|\mathrm{t}(11)=7.1, \mathrm{p}<0.001| \mathrm{p}<0.001)$. For monkey A, a significant increase in the standard deviation in reach endpoints was found for jumps towards the body $(\mathrm{t}(6)=8.6 \mathrm{p}<0.001)$ but not for jumps away from the body $(\mathrm{t}(6)=1.6 \mathrm{p}=0.2)$. Thus, reach endpoints on cursor-jump trials were more variable and biased towards the edges of the goal during wide goal reaches as compared to narrow goal reaches.

For cursor-jump trials, kinematic changes were primarily restricted to the orthogonal reach axis, which coincided with the direction that the cursor jumped (Figure 3A, B). Figure 3C shows the hand velocity after subtracting the unperturbed hand velocity ( $\Delta$ ortho reach velocity). For the narrow and wide goals, the monkey initiated a correction for the cursor jump within 123ms (blue arrow, narrow onset) and 163ms (red arrow, wide onset) of the jump onset, respectively. Differences between corrective responses for the narrow and wide goals began to differentiate $\sim 174 \mathrm{~ms}$ after the jump (solid and dashed black arrows; goal shape onset). Across recording sessions, monkey M|A initiated a correction within $113 \mid 160 \mathrm{~ms}$ and $160 \mid 173 \mathrm{~ms}$ of the cursor jumps to the narrow and wide goals, respectively. Monkeys C|T initiated a correction for the narrow goal 132|135ms after jump onset but did not reliably detect a correction for the wide goal. Hand velocity differentiated based on goal shape starting at 181|178|176|159ms (average across both directions, Figure 3D) for monkey M|A|T|C. A one-way repeated-measures ANOVA with onset type as a factor (3 levels, narrow and wide goals, and goal shape) was significant for monkey $\mathrm{M}(\mathrm{F}(2,10)=5.0, \mathrm{p}=0.03)$. Post hoc paired t-tests revealed a 
significant difference between the narrow onset and the goal-shape onset $(t(5)=4.1, p=0.02$, Bonferroni correction factor of 3$)$. The ANOVA was not significant for monkey A $(F(2,12)=2.3, p=0.14)$. Since the wide goal onsets were largely absent for monkeys $\mathrm{T}$ and $\mathrm{C}$, we instead used paired t-tests between the narrow and goal shape onsets and found onsets were not significant (monkey $\mathrm{T}|\mathrm{C} t(13)=2.0| \mathrm{t}(11)=2.0$, $\mathrm{p}=0.06 \mid \mathrm{p}=0.07)$

From monkey M, we implanted an indwelling chronic EMG system that recorded the activities of eight muscles that spanned the shoulder and elbow joints. The system allowed us to sample activity from each muscle twice and allowed us to record the same muscle across multiple recording sessions (see Methods). We recorded muscle activities across two sessions and pooled trials. Since we were largely interested in perturbation-related activity, we restricted our analysis to 14 of 16 samples that responded to the cursor jump (see perturbation-sensitive criteria in Methods). For monkey C, we recorded muscle activity using surface electrodes over the course of four sessions. We only analyzed recording days where perturbation-related activity was detected in a given muscle (number of good sessions: TLat: 3 Br: 4 Bi: 1 TLong: 3), due to the poorer signal-to-noise ratio of surface EMG than indwelling EMG and due to the variability inherent in day-to-day electrode placements. shoulder flexor) muscle aligned to the faux-jump onset from monkey $\mathrm{M}$. The temporal structure of the muscle activity was comparable between goal shapes, however, starting around the time of the fauxjump onset there was a greater increase in activity for the narrow goal than the wide goal. In contrast, the long head of the triceps (TLong, shoulder and elbow extensor) exhibited similar temporal structure and activity magnitudes for the narrow and wide goals (Figure 5B). Across the population of muscle samples for monkey M, 75\% of samples had significantly greater activities for unperturbed reaches to the narrow goal than the wide goal in the movement epoch (two-sample t-test, $\mathrm{p}<0.01$ ) with the average activity for the narrow goal being $15 \%$ greater than the activity for the wide goal (Figure 5C significant 
muscles are black filled circles). In contrast, there were no significant differences in activities for the narrow and wide goal in activity recorded from monkey $\mathrm{C}$ (Figure $5 \mathrm{C}$, grey open circles). goals (Pearson's correlation coefficient r=0.99, all muscles included from monkey M and C).

Furthermore, there was a strong temporal correlation between muscle activities for the narrow and wide goal reaches with a median correlation coefficient of 0.80 across muscles, which was significant (shuffle $r=0.16, \mathrm{p}<0.001$; Figure 5D). Thus, muscle activity was largely similar between unperturbed reaches to the narrow and wide goals with a slight bias towards greater activity for reaches to the narrow goal for monkey M.

Figures $6 \mathrm{~A}$ and $\mathrm{B}$ show the change in activities $(\triangle \mathrm{EMG})$ for PM and TLong for cursor-jump trials (monkey M). For both muscles, activity started to differentiate between jump directions starting in $<100 \mathrm{~ms}$. Activity also appeared to differentiate based on goal shape starting $\sim 100 \mathrm{~ms}$ after the jump with greater change in activity for the narrow goal than the wide goal. We averaged across muscle samples and found the population activity increased from baseline for the narrow goal starting $\sim 89 \mathrm{~ms}$ after the cursor jump (Figure 6C, bottom). However, we were unable to detect an onset for the wide goal. The population response differentiated based on the goal shape almost immediately with an onset detected at $89 \mathrm{~ms}$ (Figure 6C, top). Similar results were found with monkey $\mathrm{C}$ except that the population signals started to differentiate from baseline (116ms, Figure 6D bottom) and between goal shapes (122ms, Figure 6D top) later which may be due to the noisier nature of surface EMG. Similar trends were found when analyzing onsets of individual muscles (Figure 6E). goals in the epochs of 70-100ms, 100-125ms, 125-150ms, and 170-230ms (Figure 6F-I). For each muscle we applied a two-way ANOVA with time (levels: four epochs) and goal shape (levels: narrow and wide) as factors and found $89 \%$ of samples had a significant interaction effect between time and 
goal shape ( $\mathrm{p}<0.05$, Bonferroni correction factor of 2). Post-hoc two-sample t-tests revealed, 17\%,

$35427 \%, 61 \%$, and $78 \%$ of samples had significantly different muscle responses in the $70-100 \mathrm{~ms}, 100-$

355 125ms, 125-150ms and 170-230ms epochs, respectively ( $\mathrm{p}<0.05$, Bonferroni correction factor 4, Figure

356 6F-I filled circles). On average, the activity for the wide goal was $88 \%, 78 \%, 86 \%$ and $81 \%$ smaller

357 than the activity for the narrow goal in the $70-100 \mathrm{~ms}, 100-125 \mathrm{~ms}, 125-150 \mathrm{~ms}$, and $170-230 \mathrm{~ms}$ epochs,

358 respectively. We examined group-level responses across trial-averaged muscle responses by applying a

359 two-way repeated-measures ANOVA with time (levels: four epochs) and goal shape (levels: narrow and

360 wide) as factors. We found a significant main effect of goal shape $(F(1,17)=58.1 \mathrm{p}<0.001)$ and an

361 interaction between goal shape and time $(\mathrm{F}(3,51)=17.9, \mathrm{p}<0.001)$. Post-hoc paired t-tests confirmed

362 that responses for the narrow goal were significantly greater than for the wide goal in all epochs (70-

$363100|100-125| 125-150 \mid 170-230: \mathrm{df}=11$ (all epochs), $\mathrm{t}(11)=3.5|\mathrm{t}(11)=8.3| \mathrm{t}(11)=7.8 \mid \mathrm{t}(11)=9.7$,

$364 \mathrm{p}<0.01|\mathrm{p}<0.001| \mathrm{p}<0.001 \mid \mathrm{p}<0.001$, Bonferroni correction factor 4$)$. Collectively these results indicate

365 muscle activity in response to visual feedback of the limb differentiates based on goal shape within

$366 \sim 90-120 \mathrm{~ms}$.

368 Experiment 2: Goal redundancy and feedback responses to mechanical loads

369 We modified the above reaching task to probe feedback responses to mechanical loads. We

370 increased the size of the redundant dimension of the goal to better differentiate corrections to the

371 narrow and wide goals (Figure 1B). We also adopted a shape for the wide goal that was similar to an

372 arrowhead so that edges of the goal were in closer proximity to the monkey, thus making it easier to

373 reach on perturbation trials. For monkeys $\mathrm{M}|\mathrm{A}| \mathrm{T} \mid \mathrm{C}$, we recorded $10|11| 8 \mid 9$ behavioural sessions of the

374 animals performing the task on separate days. Again, monkeys were able to perform the task with high

375 efficiency (success rates monkeys $\mathrm{M}|\mathrm{A}| \mathrm{T} \mid \mathrm{C}$ : narrow targets=96|95|95|96\%, wide

376 targets $=97|100| 97 \mid 91 \%)$. 
one recording session. Similar to the previous task, there was more variability in the reaches to the wide goal (Figure 7B, C). Across sessions we found no significant different in endpoint position for monkey $\mathrm{M}$ and $\mathrm{T}$, whereas there was a $0.7 \mathrm{~cm}$ change in endpoint positions for monkeys $\mathrm{A}(\mathrm{t}(10)=6.1$ $\mathrm{p}<0.001)$ and $\mathrm{C}(\mathrm{t}(8)=5.8, \mathrm{p}<0.001)$. The variability of reach endpoints were $2.2|1.4| 1.4 \mid 2.3$ times

paired t-test monkey $\mathrm{M}|\mathrm{A} \mathrm{t}(9)=5.3| \mathrm{t}(10)=4.1|\mathrm{t}(7)=5.2| \mathrm{t}(8)=7.3, \mathrm{p}<0.001|\mathrm{p}=0.002| \mathrm{p}=0.001 \mid \mathrm{p}<0.001)$.

Figures $8 \mathrm{~A}$ and $\mathrm{B}$ (inset) show the unperturbed hand velocities during the same recording

session along the reach and orthogonal reach axes, respectively. In contrast to Experiment 1, there was

a small decrease in the peak hand velocity in both directions for the wide goal as compared to the

narrow goal. The hand speed was not significantly different for monkeys $\mathrm{M}$ (narrow $0.45 \mathrm{~m} / \mathrm{s}$, wide

$0.44 \mathrm{~m} / \mathrm{s}$, paired t-test $\mathrm{t}(9)=1.5, \mathrm{p}=0.2$ ) and $\mathrm{T}$ (narrow $0.38 \mathrm{~m} / \mathrm{s}$, wide $0.36 \mathrm{~m} / \mathrm{s}, \mathrm{t}(7)=2.3 \mathrm{p}=0.05$ ), however

there was a significant reduction for the wide goal for monkey A (narrow $0.52 \mathrm{~m} / \mathrm{s}$, wide $0.47 \mathrm{~m} / \mathrm{s}$,

$\mathrm{t}(10)=8.0 \mathrm{p}<0.001$ ) and a significant increase for the wide goal for monkey $\mathrm{C}$ (narrow $0.36 \mathrm{~m} / \mathrm{s}$, wide

$0.38 \mathrm{~m} / \mathrm{s}, \mathrm{t}(8)=2.6, \mathrm{p}=0.03)$.

Next, we examined how goal redundancy affected corrective responses for the unexpected mechanical loads. Figure 9A shows monkey M's hand paths on mechanical-load trials. There is a clear correction present when the monkey was reaching for the narrow goal, whereas when reaching for the wide goal monkeys exhibited greater variability and corrected less for the mechanical loads (Figure 9B, C). Across sessions, there was a greater change in reach endpoints (change relative to unperturbed trials) for the wide goal than the narrow goal (Figure 9D, F; paired t-tests: mechanical loads away from/towards body monkey $\mathrm{Mt}(9)=7.8 \mid \mathrm{t}(9)=4.9$, monkey $\mathrm{A} t(10)=9.5 \mid \mathrm{t}(10)=20$, monkey $\mathrm{T}$ 
$401 \mathrm{t}(9)=8.3|\mathrm{t}(9)=3.0, \mathrm{p}<0.001| \mathrm{p}=0.01$, monkey $\mathrm{A} \mathrm{t}(10)=5.4|\mathrm{t}(10)=4.5, \mathrm{p}<0.001| \mathrm{p}=0.001$, monkey $\mathrm{T}$

$\mathrm{t}(7)=4.8|\mathrm{t}(7)=5.4, \mathrm{p}=0.002| \mathrm{p}=0.001$, monkey $\mathrm{C} \mathrm{t}(8)=4.9|\mathrm{t}(8)=4.4, \mathrm{p}=0.001| \mathrm{p}=0.002)$.

For the mechanical-load trials, kinematic changes were primarily in the orthogonal reach axis

404 though changes could also be detected in the reach axis (Figure 8A, B). Detecting the earliest

405 kinematic correction to a mechanical load is difficult as the limb is already moving due to the

406 momentum from the load (Figure 8C). However, we could detect differences in corrections based on 407 goal shape that started at $147 \mathrm{~ms}|136 \mathrm{~ms}| 139 \mathrm{~ms} \mid 140 \mathrm{~ms}$ for monkeys M|A|T|C, respectively (Figure 8D).

$408 \quad$ For muscle activity recorded from monkey M (indwelling), we pooled recordings over the

409 course of eight behavioural sessions as the differences in the corrective responses were comparatively

410 weaker than for the cursor perturbations. All muscle samples from monkey M were included as they all

411 showed perturbation-related activity. For monkey C, we again recorded using surface electrodes over

412 the course of 7 recordings sessions and kept 6, 4, 3, and 6 recording sessions for TLat, Br, Bi, and

413 TLong, respectively.

$414 \quad$ Figure 10A and B shows the average muscle activity on unperturbed trials for PM and TLong

415 aligned to the faux-load onset (monkey M). Both muscles had similar temporal structure for the goal

416 shapes, however, for PM there was greater activity for the narrow goal than the wide goal. Across

417 muscle samples, all samples from monkey $\mathrm{M}$ had significantly greater activities for unperturbed

418 reaches to the narrow goal than the wide goal in the movement epoch (two-sample t-test, $\mathrm{p}<0.01)$ with

419 the average activity for the wide goal being 17\% smaller than the activity for the narrow goal (Figure

420 10C significant muscles are filled black circles). In contrast, no muscle samples collected from

421 monkey $\mathrm{C}$ were significantly different between the narrow and wide goals (grey circles). The muscle

422 activity magnitudes were highly correlated between the two goal shapes for both monkeys (Pearson's

423 correlation coefficient across all muscles $\mathrm{r}=0.98$ ) and there were was a strong temporal correlation 
between activities for the narrow and wide goals (Figure 10D, median r=0.891), and the distribution across muscles was shifted more to the right than the shuffled distribution (Inset, $\mathrm{r}=0.49, \mathrm{p}<0.001$ ).

increased from baseline starting at $62 \mathrm{~ms}$ and $64 \mathrm{~ms}$ for the narrow and wide goals, respectively (Figure shape (levels: narrow and wide) to the trial-averaged muscle responses. We found a significant main 441 effect of goal shape $(\mathrm{F}(1,19)=22.3 \mathrm{p}<0.001)$ and an interaction between goal shape and time $442(\mathrm{~F}(3,57)=17.8, \mathrm{p}<0.001)$. Post-hoc paired t-tests confirmed that responses for the narrow goal were 443 significantly greater than for the wide goal in the $75-100 \mathrm{~ms}(\mathrm{t}(19)=3.2, \mathrm{p}=0.004)$ and $120-180 \mathrm{~ms}$ $444(\mathrm{t}(19)=5.1, \mathrm{p}<0.001)$ epochs, but not significantly different in the earlier epochs $(20-50 \mathrm{~ms} t(19)=0.3$ 
$\underline{\text { Discussion }}$

Exploiting redundancies is an important feature of many motor control theories including OFC 448 (Bernstein, 1967; Scholz et al., 2000; Todorov and Jordan, 2002; Latash, 2012). Several studies

449 demonstrate how humans are capable of exploiting redundancies during motor actions (Todorov and 450 Jordan, 2002; Diedrichsen, 2007; Mutha and Sainburg, 2009; Knill et al., 2011; Dimitriou et al., 2012;

451 Nashed et al., 2012, 2014; Cluff and Scott, 2015). Here, we demonstrate that monkeys are able to 452 exploit the spatial redundancy of a goal as previously observed in humans. Specifically, monkeys 453 exhibited greater variability in their reach endpoints when reaching to a more spatially redundant goal. 454 Rapid corrective responses to limb perturbations were also greatly attenuated to exploit goal 455 redundancy with observable changes in muscle activity starting in $<100 \mathrm{~ms}$.

Although it is impossible to know for certain whether the monkey recognized that they could reach anywhere on the wide targets, there are several lines of evidence that indicate monkeys exploited goal redundancy similar to humans and OFC models. First, OFC predicts that trial-by-trial variability even on unperturbed trials should be larger for goals with greater spatial redundancy with variability constrained along the redundant axis of the goal (Knill et al., 2011; Nashed et al., 2012). Previous studies in humans (Knill et al., 2011; Nashed et al., 2012; Cross et al., 2019) and the current study in monkeys demonstrate similar OFC-like structure with variability growing throughout the duration of the reach and culminating in greater variability in the reach endpoints along the redundant axis of the 464 goal.

Second, OFC models predict that corrections to external perturbations should be smaller for more spatially redundant goals provided the perturbation is along the redundant axis. Previous studies demonstrate that humans share this feature in their corrective responses to visual and mechanical perturbations (Knill et al., 2011; Nashed et al., 2012; Cross et al., 2019). Here, we demonstrate monkeys also show smaller corrective responses when reaching for the wide goal. This was evident as 
a $2-4 \mathrm{~cm}$ shift in the reach endpoints on perturbation trials from where monkeys were reaching on

471 unperturbed trials. In contrast, there was $<1 \mathrm{~cm}$ shift in endpoints when monkeys were reaching for the

472 narrow goal on perturbation trials. Endpoints on perturbation trials were also more variable when

473 reaching for the wide goal indicating monkeys did not simply learn to reach for a particular goal

474 location on perturbation trials. Collectively, these results argue that that monkeys in the present study

475 understood goal redundancy with behaviour comparable to human performance and did not simply

476 learn an arbitrary mapping between sensory stimuli and behavioural response that mimicked the

477 expected behaviour.

Monkeys also generated muscle activity patterns to the mechanical loads that were similar to

the OFC model and humans. OFC models predict an initial increase in control output in response to the

mechanical load regardless of goal shape reflecting that the controller must counteract the external load

to stabilize the limb (Nashed et al., 2012). Control output differentiates based on goal shape later with

482 greater activity for the narrow goal to generate the necessary kinematic correction. Muscle activity

483 evoked by the mechanical loads exhibit similar patterns as the control output with an initial increase

484 starting at $\sim 50 \mathrm{~ms}$ followed by differentiation based on goal shape starting at $\sim 70 \mathrm{~ms}$ in both humans

485 (Nashed et al., 2012) and monkeys (present study). Other studies have also found humans and monkeys

486 exhibit similar timing for when corrective responses to mechanical loads are modulated by different

487 contexts including for limb physics (Kurtzer et al., 2008; Pruszynski et al., 2011), task instruction

488 (Hammond, 1956; Evarts and Tanji, 1976; Pruszynski et al., 2008, 2014; Omrani et al., 2014), and

489 adaptation (Cluff and Scott, 2013; Maeda et al., 2018, 2020) which all start 60-70ms after the onset of

490 the load. This similarity highlights how monkeys are a useful model to investigate the neural circuits

491 that underlie flexible feedback processing during motor actions.

492 Our results contrast with previous findings by Bizzi and colleagues (1982, 1984) who found

493 monkeys correct back to the original trajectory when encountering an assistive mechanical load. One 
494 possible reason is that monkeys may have learned an implicit timing constraint for when the arm

495 should arrive at the goal and thus resisted the applied load to prevent arriving too early. Indeed, humans 496 also show similar corrections but only when given a timing constraint (Cluff and Scott, 2015). reaches to the narrow goal than the wide goal, particularly for monkey M. Presumably, this increased activity was also present on mechanical-load trials and poses a potential problem for interpreting onsets to mechanical loads due to a gain-scaling effect where muscle activity evoked by a load scales with the size of the background muscle activity (Marsden et al., 1976; Bedingham and Tatton, 1984; Matthews, 1986; Stein et al., 1995; Pruszynski et al., 2009). Thus, greater muscle activity for the narrow goal following a mechanical load could simply reflect a gain-scaling effect. However, we believe this is unlikely as the effect of gain scaling typically only influences muscle activity within 20-50ms after an applied load (Pruszynski et al., 2009; Nashed et al., 2012) whereas in our study activity differentiated based on goal shape at $\sim 70 \mathrm{~ms}$. Second, we did not detect a significant difference in muscle activity for monkey $\mathrm{C}$ on unperturbed trials using surface electrodes and still found similar muscle timing. Studies highlight that primary motor cortex (M1) is involved with generating flexible muscle responses to sensory feedback. M1 receives rich proprioceptive feedback with responses that start 510 within $20 \mathrm{~ms}$ of an applied load (Conrad et al., 1974, 1975; Wolpaw, 1980; Fromm et al., 1984; 511 Bauswein et al., 1991; Picard and Smith, 1992; Herter et al., 2009; Takei et al., 2018; Heming et al., 512 2019; Cross et al., 2020, 2021). Importantly, proprioceptive feedback responses in M1 are modulated 513 by several behavioural factors including limb physics (Pruszynski et al., 2011), prior instruction (Evarts 514 and Tanji, 1976; Pruszynski et al., 2014), and task engagement (Omrani et al., 2014) within 50ms of an 515 applied load. The flexible responses in M1 preceded the corresponding change in muscle activity by 516 10ms consistent with the conduction delay between M1 and the periphery (Cheney and Fetz, 1984; 517 Lemon et al., 1986). Thus, if M1 is involved with generating proprioceptive feedback responses that 
518 exploits goal redundancy than M1 activity reflecting goal redundancy should emerge $\sim 60 \mathrm{~ms}$ after the 519 load onset.

520 However, it is likely that other brain areas also contribute to generating muscle responses that 521 exploit goal redundancy. Premotor cortex, somatosensory cortex, parietal area 5and the cerebellum all 522 project to M1 (Jones et al., 1978; Porter and Lemon, 1993; Dea et al., 2016) and rapidly respond to 523 proprioceptive feedback within $<70 \mathrm{~ms}$ with activity patterns that are context dependent (Wolpaw, 1980; 524 Lamarre et al., 1983; Strick, 1983; Chapman et al., 1984; Pruszynski et al., 2011, 2014; London and 525 Miller, 2012; Omrani et al., 2014, 2016). However, context is not homogenously shared across these 526 areas as premotor cortex, M1 and cerebellum exhibit activity patterns consistent with implementing a 527 control policy whereas somatosensory and parietal areas exhibit patterns of activity consistent with 528 state estimation (Strick, 1983; Omrani et al., 2016). These results are also supported by a recent study 529 examining temporary inactivation of a subset of these areas (Takei et al., 2021). Thus, given that goal 530 redundancy is a property of the control policy, we predict context-dependent responses will emerge first 531 in premotor cortex, M1 and the cerebellum.

532 Monkeys also generated muscle activity patterns to the cursor jumps that were similar to 533 humans and OFC models. Unlike mechanical loads, cursor jumps do not require the controller to 534 counteract an external load as the disturbance is only a kinematic error. Thus, control output of the 535 OFC model should be unaffected by the cursor jump when reaching for the wide goal. Similarly, 536 muscle activity of humans (Cross et al., 2019) and monkeys are largely unchanged by a cursor jump 537 when reaching to a wide goal resulting in activity that differentiates based on goal redundancy $\sim 90 \mathrm{~ms}$ 538 after a cursor jump. This differentiation is also unlikely due to a gain-scaling effect as background 539 muscle activity does not appear to affect correction strength for visual perturbations (Franklin et al., 540 2017). 
It is less clear how visual feedback is processed by fronto-parietal circuits and how behavioural

542 context influences visual processing in these areas. A common assumption is that visual feedback is

543 processed by posterior parietal cortex which is then sent towards frontal circuits including M1 and

544 premotor cortex (Goodale and Milner, 1992; Desmurget et al., 1999; Pisella et al., 2000; Gaveau et al.,

545 2014). Thus, consistent with OFC visual feedback is processed initially by circuits involved with state

546 estimation followed by circuits involved with implementing the control policy. However, there is

547 evidence that visual feedback responses arrive first in premotor cortex (50-70ms) followed by M1 (70-

548 100) and finally parietal area 5 (Cisek and Kalaska, 2005; Archambault et al., 2011; Ames et al., 2014;

549 Stavisky et al., 2017; Cross et al., 2021). Thus, premotor cortex may generate the earliest muscle

550 response to visual feedback rather than $\mathrm{M} 1$.

Alternatively the superior colliculus may be involved with generating rapid motor responses to

552 visual feedback (Alstermark et al., 1987; Day and Brown, 2001; Pruszynski et al., 2010; Corneil and

553 Munoz, 2014; Day, 2014; Cross et al., 2019; Kozak et al., 2019). Activity in the superior colliculus

554 correlates with muscle activity of the upper arm during reaching (Werner, 1993; Werner et al., 1997;

555 Stuphorn et al., 1999) and stimulation of the superior colliculus can evoke reaching-like behaviour

556 (Philipp and Hoffmann, 2014). Further investigations are needed to elucidate the underlying neural

557 circuits involved with generating rapid visual responses for which our behavioural task could be 558 invaluable. 
References

560

561

562

563

564

565

566

567

568

569

570

571

572

573

574

575

576

577

578

579

580

581

582

583

584

585

586

587

588

589

590

591

Alstermark B, Gorska T, Lundberg A, Pettersson L-G, Walkowska M (1987) Effect of different spinal cord lesions on visually guided switching of target-reaching in cats. Neurosci Res 5:63-67.

Ames KC, Ryu SI, Shenoy KV (2014) Neural Dynamics of Reaching following Incorrect or Absent Motor Preparation. Neuron 81:438-451.

Archambault PS, Ferrari-Toniolo S, Battaglia-Mayer A (2011) Online control of hand trajectory and evolution of motor intention in the parietofrontal system. J Neurosci Off J Soc Neurosci $31: 742-752$.

Bauswein E, Fromm C, Werner W, Ziemann U (1991) Phasic and tonic responses of premotor and primary motor cortex neurons to torque changes. Exp Brain Res 86 Available at: http://link.springer.com/10.1007/BF00228953 [Accessed June 15, 2020].

Bedingham W, Tatton WG (1984) Dependence of EMG Responses Evoked by Imposed Wrist Displacements on Pre-existing Activity in the Stretched Muscles. Can J Neurol Sci J Can Sci Neurol 11:272-280.

Bernstein N (1967) The co-ordination and regulation of movements. Pergamon-Press.

Bizzi E, Accornero N, Chapple W, Hogan N (1982) Arm trajectory formation in monkeys. Exp Brain Res 46:139-143.

Bizzi E, Accornero N, Chapple W, Hogan N (1984) Posture control and trajectory formation during arm movement. J Neurosci 4:2738-2744.

Bonnet M (1983) Anticipatory changes of long-latency stretch responses during preparation for directional hand movements. Brain Res 280:51-62.

Chapman CE, Spidalieri G, Lamarre Y (1984) Discharge properties of area 5 neurones during arm movements triggered by sensory stimuli in the monkey. Brain Res 309:63-77.

Cheney PD, Fetz EE (1984) Corticomotoneuronal cells contribute to long-latency stretch reflexes in the rhesus monkey. J Physiol 349:249-272.

Cisek P, Kalaska JF (2005) Neural Correlates of Reaching Decisions in Dorsal Premotor Cortex: Specification of Multiple Direction Choices and Final Selection of Action. Neuron 45:801-814.

Cluff T, Scott SH (2013) Rapid Feedback Responses Correlate with Reach Adaptation and Properties of Novel Upper Limb Loads. J Neurosci 33:15903-15914.

Cluff T, Scott SH (2015) Apparent and Actual Trajectory Control Depend on the Behavioral Context in Upper Limb Motor Tasks. J Neurosci 35:12465-12476.

Conrad B, Matsunami K, Meyer-Lohmann J, Wiesendanger M, Brooks VB (1974) Cortical load compensation during voluntary elbow movements. Brain Res 71:507-514. 
Conrad B, Meyer-Lohmann J, Matsunami K, Brooks VB (1975) Precentral unit activity following torque pulse injections into elbow movements. Brain Res 94:219-236.

Corneil BD, Munoz DP (2014) Overt Responses during Covert Orienting. Neuron 82:1230-1243.

Corneil BD, Olivier E, Munoz DP (2004) Visual responses on neck muscles reveal selective gating that prevents express saccades. Neuron 42:831-841.

Crago PE, Houk JC, Hasan Z (1976) Regulatory actions of human stretch reflex. J Neurophysiol 39:925-935.

Cross KP, Cluff T, Takei T, Scott SH (2019) Visual Feedback Processing of the Limb Involves Two Distinct Phases. J Neurosci 39:6751-6765.

Cross KP, Cook DJ, Scott SH (2021) Convergence of proprioceptive and visual feedback on neurons in primary motor cortex. bioRxiv:2021.05.01.442274.

Cross KP, Heming EA, Cook DJ, Scott SH (2020) Maintained Representations of the Ipsilateral and Contralateral Limbs during Bimanual Control in Primary Motor Cortex. J Neurosci 40:67326747.

Day BL (2014) Subcortical Visuomotor Control of Human Limb Movement. In: Progress in Motor Control (Levin MF, ed), pp 55-68. New York, NY: Springer New York. Available at: http://link.springer.com/10.1007/978-1-4939-1338-1_5 [Accessed September 25, 2017].

Day BL, Brown P (2001) Evidence for subcortical involvement in the visual control of human reaching. Brain 124:1832-1840.

de Brouwer AJ, Jarvis T, Gallivan JP, Flanagan JR (2017) Parallel Specification of Visuomotor Feedback Gains during Bimanual Reaching to Independent Goals. eneuro 4:ENEURO.002617.2017.

Dea M, Hamadjida A, Elgbeili G, Quessy S, Dancause N (2016) Different Patterns of Cortical Inputs to Subregions of the Primary Motor Cortex Hand Representation in Cebus apella. Cereb Cortex 26:1747-1761.

Desmurget M, Epstein CM, Turner RS, Prablanc C, Alexander GE, Grafton ST (1999) Role of the posterior parietal cortex in updating reaching movements to a visual target. Nat Neurosci 2:563-567.

Diedrichsen J (2007) Optimal Task-Dependent Changes of Bimanual Feedback Control and Adaptation. Curr Biol 17:1675-1679.

Dimitriou M, Franklin DW, Wolpert DM (2012) Task-dependent coordination of rapid bimanual motor responses. J Neurophysiol 107:890-901.

Dimitriou M, Wolpert DM, Franklin DW (2013) The Temporal Evolution of Feedback Gains Rapidly Update to Task Demands. J Neurosci 33:10898-10909. 
Evarts EV, Tanji J (1976) Reflex and intended responses in motor cortex pyramidal tract neurons of monkey. J Neurophysiol 39:1069-1080.

Flash T, Hogan N (1985) The coordination of arm movements: an experimentally confirmed mathematical model. J Neurosci 5:1688-1703.

Franklin DW, Wolpert DM (2008) Specificity of Reflex Adaptation for Task-Relevant Variability. J Neurosci 28:14165-14175.

Franklin S, Wolpert DM, Franklin DW (2017) Rapid visuomotor feedback gains are tuned to the task dynamics. J Neurophysiol:2711-2726.

Fromm C, Wise SP, Evarts EV (1984) Sensory response properties of pyramidal tract neurons in the precentral motor cortex and postcentral gyrus of the rhesus monkey. Exp Brain Res 54 Available at: http://link.springer.com/10.1007/BF00235829 [Accessed June 24, 2020].

Gaveau V, Pisella L, Priot A-E, Fukui T, Rossetti Y, Pélisson D, Prablanc C (2014) Automatic online control of motor adjustments in reaching and grasping. Neuropsychologia 55:25-40.

Goodale MA, Milner AD (1992) Separate visual pathways for perception and action. Trends Neurosci 15:20-25.

Gu C, Wood DK, Gribble PL, Corneil BD (2016) A Trial-by-Trial Window into Sensorimotor Transformations in the Human Motor Periphery. J Neurosci 36:8273-8282.

Hammond P (1956) The influence of prior instruction to the subject on an apparently involuntary neuro-muscular response. J Physiol 132:17-18P.

Heming EA, Cross KP, Takei T, Cook DJ, Scott SH (2019) Independent representations of ipsilateral and contralateral limbs in primary motor cortex. eLife 8:e48190.

Herter TM, Korbel T, Scott SH (2009) Comparison of Neural Responses in Primary Motor Cortex to Transient and Continuous Loads During Posture. J Neurophysiol 101:150-163.

Jones EG, Coulter JD, Hendry SHC (1978) Intracortical connectivity of architectonic fields in the somatic sensory, motor and parietal cortex of monkeys. J Comp Neurol 181:291-347.

Keyser J, Medendorp WP, Selen LPJ (2017) Task-dependent vestibular feedback responses in reaching. J Neurophysiol 118:84-92.

Keyser J, Ramakers REFS, Medendorp WP, Selen LPJ (2019) Task-dependent responses to muscle vibration during reaching. Eur J Neurosci 49:1477-1490.

Knill DC, Bondada A, Chhabra M (2011) Flexible, Task-Dependent Use of Sensory Feedback to Control Hand Movements. J Neurosci 31:1219-1237.

Kozak RA, Kreyenmeier P, Gu C, Johnston K, Corneil BD (2019) Stimulus-Locked Responses on Human Upper Limb Muscles and Corrective Reaches Are Preferentially Evoked by Low Spatial Frequencies. eNeuro 6 Available at: https://www.ncbi.nlm.nih.gov/pmc/articles/PMC6751371/ [Accessed April 6, 2020]. 
Kurtzer IL, Pruszynski JA, Scott SH (2008) Long-Latency Reflexes of the Human Arm Reflect an Internal Model of Limb Dynamics. Curr Biol 18:449-453.

Lamarre Y, Spidalieri G, Chapman CE (1983) A comparison of neuronal discharge recorded in the sensori-motor cortex, parietal cortex and dentate nucleus of the monkey during arm movements triggered by light, sound or somesthetic stimuli. Exp Brain Res 49:140-156.

Latash ML (2012) The Bliss of Motor Abundance. Exp Brain Res 217:1-5.

Lee RG, Tatton WG (1975) Motor Responses to Sudden Limb Displacements in Primates with Specific CNS Lesions and in Human Patients with Motor System Disorders. Can J Neurol Sci J Can Sci Neurol 2:285-293.

Lemon RN, Mantel GW, Muir RB (1986) Corticospinal facilitation of hand muscles during voluntary movement in the conscious monkey. J Physiol 381:497-527.

London BM, Miller LE (2012) Responses of somatosensory area 2 neurons to actively and passively generated limb movements. J Neurophysiol 109:1505-1513.

Lowrey CR, Nashed JY, Scott SH (2017) Rapid and flexible whole body postural responses are evoked from perturbations to the upper limb during goal-directed reaching. J Neurophysiol 117:10701083.

Maeda RS, Cluff T, Gribble PL, Pruszynski JA (2018) Feedforward and Feedback Control Share an Internal Model of the Arm's Dynamics. J Neurosci 38:10505-10514.

Maeda RS, Kersten R, Pruszynski JA (2020) Shared internal models for feedforward and feedback control of arm dynamics in non-human primates Smith Y, ed. Eur J Neurosci Available at: https://onlinelibrary.wiley.com/doi/10.1111/ejn.15056 [Accessed February 9, 2021].

Marsden CD, Merton PA, Morton HB (1976) Servo action in the human thumb. J Physiol 257:1-44.

Matthews PB (1986) Observations on the automatic compensation of reflex gain on varying the preexisting level of motor discharge in man. J Physiol 374:73-90.

Mutha PK, Sainburg RL (2009) Shared Bimanual Tasks Elicit Bimanual Reflexes During Movement. J Neurophysiol 102:3142-3155.

Nashed JY, Crevecoeur F, Scott SH (2012) Influence of the behavioral goal and environmental obstacles on rapid feedback responses. J Neurophysiol 108:999-1009.

Nashed JY, Crevecoeur F, Scott SH (2014) Rapid Online Selection between Multiple Motor Plans. J Neurosci 34:1769-1780.

Omrani M, Murnaghan CD, Pruszynski JA, Scott SH (2016) Distributed task-specific processing of somatosensory feedback for voluntary motor control. eLife 5:e13141.

Omrani M, Pruszynski JA, Murnaghan CD, Scott SH (2014) Perturbation-evoked responses in primary motor cortex are modulated by behavioral context. J Neurophysiol 112:2985-3000. 
Philipp R, Hoffmann K-P (2014) Arm Movements Induced by Electrical Microstimulation in the Superior Colliculus of the Macaque Monkey. J Neurosci 34:3350-3363.

Picard N, Smith AM (1992) Primary motor cortical responses to perturbations of prehension in the monkey. J Neurophysiol 68:1882-1894.

Pisella L, Gréa H, Tilikete C, Vighetto A, Desmurget M, Rode G, Boisson D, Rossetti Y (2000) An 'automatic pilot' for the hand in human posterior parietal cortex: toward reinterpreting optic ataxia. Nat Neurosci 3:729-736.

Porter R, Lemon R (1993) Corticospinal function and voluntary movement. Clarendon Press.

Pruszynski JA, Johansson RS, Flanagan JR (2016) A Rapid Tactile-Motor Reflex Automatically Guides Reaching toward Handheld Objects. Curr Biol 26:788-792.

Pruszynski JA, King GL, Boisse L, Scott SH, Flanagan JR, Munoz DP (2010) Stimulus-locked responses on human arm muscles reveal a rapid neural pathway linking visual input to arm motor output: Visual responses on human arm muscles. Eur J Neurosci 32:1049-1057.

Pruszynski JA, Kurtzer I, Lillicrap TP, Scott SH (2009) Temporal Evolution of “Automatic GainScaling." J Neurophysiol 102:992-1003.

Pruszynski JA, Kurtzer I, Nashed JY, Omrani M, Brouwer B, Scott SH (2011) Primary motor cortex underlies multi-joint integration for fast feedback control. Nature 478:387-390.

Pruszynski JA, Kurtzer I, Scott SH (2008) Rapid Motor Responses Are Appropriately Tuned to the Metrics of a Visuospatial Task. J Neurophysiol 100:224-238.

Pruszynski JA, Omrani M, Scott SH (2014) Goal-Dependent Modulation of Fast Feedback Responses in Primary Motor Cortex. J Neurosci 34:4608-4617.

Scholz JP, Schöner G (1999) The uncontrolled manifold concept: identifying control variables for a functional task. Exp Brain Res 126:289-306.

Scholz JP, Schöner G, Latash ML (2000) Identifying the control structure of multijoint coordination during pistol shooting. Exp Brain Res 135:382-404.

Scott SH (1999) Apparatus for measuring and perturbing shoulder and elbow joint positions and torques during reaching. J Neurosci Methods 89:119-127.

Scott SH (2004) Optimal feedback control and the neural basis of volitional motor control. Nat Rev Neurosci 5:532-546.

Sporns O, Edelman GM (1993) Solving Bernstein's Problem: A Proposal for the Development of Coordinated Movement by Selection. Child Dev 64:960-981.

Stavisky SD, Kao JC, Ryu SI, Shenoy KV (2017) Motor Cortical Visuomotor Feedback Activity Is Initially Isolated from Downstream Targets in Output-Null Neural State Space Dimensions. Neuron 95:195-208.e9. 
752

753

754

755

Stein RB, Hunter IW, Lafontaine SR, Jones LA (1995) Analysis of short-latency reflexes in human elbow flexor muscles. J Neurophysiol 73:1900-1911.

Strick PL (1983) The influence of motor preparation on the response of cerebellar neurons to limb displacements. J Neurosci 3:2007-2020.

Stuphorn V, Hoffmann K-P, Miller LE (1999) Correlation of primate superior colliculus and reticular formation discharge with proximal limb muscle activity. J Neurophysiol 81:1978-1982.

Takei T, Crevecoeur F, Herter TM, Cross KP, Scott SH (2018) Correlations Between Primary Motor Cortex Activity with Recent Past and Future Limb Motion During Unperturbed Reaching. J Neurosci 38:7787-7799.

Takei T, Lomber SG, Cook DJ, Scott SH (2021) Transient deactivation of dorsal premotor cortex or parietal area 5 impairs feedback control of the limb in macaques. Curr Biol 1:1-12.

Todorov E, Jordan MI (2002) Optimal Feedback Control as a Theory of Motor Coordination. Nat Neurosci 5:1226-1235.

Weiler J, Gribble PL, Pruszynski JA (2015) Goal-dependent modulation of the long-latency stretch response at the shoulder, elbow, and wrist. J Neurophysiol 114:3242-3254.

Weiler J, Saravanamuttu J, Gribble PL, Pruszynski JA (2016) Coordinating long-latency stretch responses across the shoulder, elbow, and wrist during goal-directed reaching. J Neurophysiol 116:2236-2249.

Werner W (1993) Neurons in the primate superior colliculus are active before and during arm movements to visual targets. Eur J Neurosci 5:335-340.

Werner W, Hoffmann K-P, Dannenberg S (1997) Anatomical distribution of arm-movement-related neurons in the primate superior colliculus and underlying reticular formation in comparison with visual and saccadic cells: Exp Brain Res 115:206-216.

Wolpaw JR (1980) Amplitude of responses to perturbation in primate sensorimotor cortex as a function of task. J Neurophysiol 44:1139-1147. 


\section{Figure Legends}

Figure 1. Experimental set-up. A) Monkeys placed their arm inside a robotic exoskeleton and were trained to reach from a starting position (Start Target) to either a narrow target (top) or wide target (bottom). Inset: on random trials, the visual feedback of the hand (cursor) jumped orthogonally by 3$4 \mathrm{~cm}$ (see Methods). B) Same as A) showing the configuration of the targets during the mechanical perturbation experiment. Inset: on random trials, mechanical loads were applied to the limb that displaced the limb orthogonally.

Figure 2. Example kinematics on unperturbed trials for the cursor-jump variant of task. A) Top: Hand paths from one session for Monkey M to the narrow (left) and wide (right) goals. Arrow denotes the direction of reach. Dashed line on the wide goal denotes the redundant axis. Bottom: Reach endpoints and the $95 \%$ confidence ellipse. B) Standard deviations of the hand position across trials for the narrow and wide goal reaches. C) Reach endpoint histograms for reaches to the narrow and wide goals from the same session as A). Zero denotes the middle of the redundant axis (A, middle of dashed line). Arrows denote the means of the distributions. D) The mean endpoint position for the narrow and wide goals across all recording sessions. Yellow diamonds denote the means across sessions. E) Same as D) for standard deviation of the endpoint position. Hand paths on cursor-jump trials from the same session as A). ** $\mathrm{p}<0.01, * * * \mathrm{p}<0.001$.

Figure 3. Example hand velocity profiles for the cursor-jump variant of task. A) The hand velocity along the reach axis (see Figure 1) for the narrow and wide goals from the same recording session as Figure 2A). Velocity was aligned to the jump onset. Inset shows the unperturbed reaches to the narrow and wide goals. Unp Narrow and Unp Wide: Unperturbed reaches to the narrow and wide goals, respectively. CJ Narrow and CJ Wide: Cursor jump trials for reaches to the narrow and wide goals, respectively. Solid and dashed lines denote cursor jumps away from the body and towards the body, respectively. B) Same as A) for the hand velocity along the orthogonal reach axis. C) The change in the hand velocity on cursor-jump trials for the narrow and wide goals. Same recording session as A). Blue and red arrows denote the earliest corrective onset for the narrow and wide goals, respectively. Black arrows denote when corrective movements differentiate based on goal shape. D) The earliest corrective onsets for the narrow (blue) and wide goals (red) along with the onsets for when corrections differentiated based on goal shape (black) across sessions. Yellow diamonds are the means across sessions. $* \mathrm{p}<0.05$.

Figure 4. Example kinematics on cursor-jump trials. A) Top: Hand paths from Monkey M for cursor-jump trials. Same session as Figure 2A. Bottom: Reach endpoints and the 95\% confidence ellipse. Solid and dashed lines denote cursor jumps away from the body and towards the body, respectively. B) Standard deviation of the hand position across trials for the narrow and wide goal reaches. C) The change in reach endpoint histograms (change relative to unperturbed reach trials). Zero denotes the mean of the reach endpoints on unperturbed trials (Figure $2 \mathrm{C}$ arrows). Arrows denote the means of the distributions. D) The mean change in reach endpoint for jumps away from the body across all recording sessions. E) Same as D) for the standard deviation in reach endpoints. F-G) Same as D-E) for jumps towards the body. $* * \mathrm{p}<0.01,{ }^{* * *} \mathrm{p}<0.001$.

Figure 5. Muscle activity during unperturbed reaches to the narrow and wide goals for the cursor-variant of task. A) Average activity of the pectoralis major muscle for reaches to the narrow and wide goals. Activity are aligned to faux jump onset. B) Same as A) for the long head of the triceps. C) Comparison of the mean narrow and wide goal activities across muscles inside the 400ms epoch 
804

805

806

807

808

809

810

811

812

813

814

815

816

817

818

819

820

821

822

823

824

825

826

827

828

829

830

831

832

833

834

835

836

837

838

839

840

841

842

843

844

845

846

847

848

849

850

851

centered on the faux jump onset. 'r' denotes Pearson's correlation coefficient. Monkey M and C are denoted by the black and grey markers. Filled circles denote muscles with significantly different activities for the narrow and wide goals. D) Cumulative sum of the temporal correlation coefficients between the narrow and wide goal activities across muscles (Monkey M and C pooled). Same $400 \mathrm{~ms}$ epoch as C). Inset shows the median correlation coefficient from the shuffled distribution (mean \pm standard deviation) and the observed median coefficient.

Figure 6. Muscle activity in response to the cursor jumps. A) The change in activity for the pectoralis major muscle in response to the cursor jumps when reaching for the narrow and wide goals. B) Same as A) for the long head of the triceps. C) Group average change in muscle activity to the cursor jumps for the narrow (blue, bottom) and wide goals (red, bottom). The resulting difference signal between the activities for the narrow and wide goals is shown in the black trace (top). Muscle activities were averaged across their preferred directions. Blue and black arrows denote when a significant increase in activity from baseline started for the narrow goal reaches and difference signal, respectively. No onset was detected for the wide goal reaches. D) Same as C) for Monkey C. E) Onsets for individual muscles presented as a cumulative sum. Numbers in brackets reflect the number of muscle samples with a detectable onset. Muscles recorded from Monkey M and C pooled. F) Comparison between the absolute change in muscle activities for the narrow and wide goals in the 70$100 \mathrm{~ms}$ epoch. Muscles recorded from Monkey M and C are denoted in the black and grey markers. Yellow circles and bars denote the means and standard deviations for each monkey. Filled circles denote muscle samples that had significantly different activities for the narrow and wide goals. G-I) same as F) except for the 100-125ms (G), 125-150ms (H) and 170-230ms (I) epochs.

\section{Figure 7. Example kinematics for unperturbed trials for the mechanical-load variant of task. A)} Hand paths from one session for Monkey M to the narrow (left) and wide (right) goals. Dashed line on the wide goal denotes the redundant axis. B) Standard deviations of the hand position across trials for the narrow and wide goal reaches. C) Reach endpoint histograms for reaches to the narrow and wide goals from the same session as A). Zero denotes the middle of the redundant axis (A, middle of dashed line). Arrows denote the means of the distributions. D) The mean endpoint position for the narrow and wide goals across all recording sessions from both monkeys. Yellow diamonds denote the means across sessions. E) Same as D) for standard deviation of the endpoint position. ${ }^{* *} \mathrm{p}<0.01, * * * \mathrm{p}<0.001$.

Figure 8. Example hand velocity profiles for the mechanical-load variant of task. A) The hand velocity along the reach axis for the narrow and wide goals from the same recording session as Figure 7A). Velocity was aligned to the load onset. Inset shows the unperturbed reaches to the narrow and wide goals. Unp Narrow and Unp Wide: Unperturbed reaches to the narrow and wide goals, respectively. MP Narrow and MP Wide: Mechanical load trials for reaches to the narrow and wide goals, respectively. Solid and dashed lines denote mechanical loads away from the body and towards the body, respectively. B) Same as A) for the hand velocity along the orthogonal reach axis. C) The change in the hand velocity on mechanical-load trials for the narrow and wide goals. D) Time when corrections differentiated based on goal shape across sessions. Yellow diamonds are the means across sessions.

Figure 9. Example kinematics on mechanical-load variant of task. A) Top: Hand paths from Monkey M for mechanical-load trials. Same session as Figure 8A. Bottom: Reach endpoints and the 95\% confidence ellipse. Solid and dashed lines denote mechanical load away from the body and towards the body, respectively. B) Standard deviations of the hand position across trials for the narrow and wide goal reaches. C) The change in reach endpoint histograms (change relative to unperturbed 
852

853

854

855

856

857

858

859

860

861

862

863

864

865

866

867

868

869

870

871

872

873

874

875

876

877

878

879

880

881

882

883

884 reach trials). Zero denotes the mean of the reach endpoints on unperturbed trials (Figure 8C arrows). Arrows denote the means of the distributions. D) The mean change in reach endpoint for loads away from the body across all recording sessions. E) Same as D) for the standard deviation in reach endpoints. F-G) Same as D-E) for loads towards the body. ** $\mathrm{p}<0.01$, *** $\mathrm{p}<0.001$.

Figure 10. Muscle activity during unperturbed reaches to the narrow and wide goals for the mechanical-load variant of task. A) Average activity of the pectoralis major muscle for reaches to the narrow and wide goals. Activity are aligned to faux-load onset. B) Same as A) for the long head of the triceps. C) Comparison of the mean narrow and wide goal activities across muscles inside the $400 \mathrm{~ms}$ epoch centered on the faux-load onset. 'r' denotes Pearson's correlation coefficient. Monkey M and C are denoted by the black and grey markers. Filled circles denote muscles with significantly different activities for the narrow and wide goals. D) Cumulative sum of the temporal correlation coefficients between the narrow and wide goal activities across muscles (muscles from Monkey $\mathrm{M}$ and $\mathrm{C}$ pooled). Same 400ms epoch as C). Inset shows the median correlation coefficient from the shuffled distribution (mean \pm standard deviation) and the observed median coefficient.

Figure 11. Muscle activity in response to the mechanical loads. A) The change in activity for the pectoralis major muscle in response to the mechanical loads when reaching for the narrow and wide goals. B) Same as A) for the long head of the triceps. C) Group average change in muscle activity for the mechanical loads for the narrow (blue, bottom) and wide goals (red, bottom). The resulting difference signal between the activities for the narrow and wide goals is shown in the black trace (top). Muscle activities were averaged across their preferred directions. Blue and red arrows denote when a significant increase in activity from baseline $(500 \mathrm{~ms}$ before mechanical load) started for the narrow and wide goal reaches, respectively. Black arrow denotes when a significant increase in activity from baseline started for the difference signal. D) Same as C) for Monkey C. E) Onsets for individual muscles presented as a cumulative sum. Numbers in brackets reflect the number of muscle samples with a detectable onset. Muscles from Monkey $\mathrm{M}$ and $\mathrm{C}$ were pooled. F) Comparison between the absolute change in muscle activities for the narrow and wide goals in the 20-50ms epoch. Muscles recorded from Monkey $\mathrm{M}$ and $\mathrm{C}$ are denoted in the black and grey markers. Yellow circles and bars denote the mean and standard deviation for each monkey. Filled circles denote muscle samples that had significantly different activities for the narrow and wide goals. G-I) same as F) except for the 5075ms (G), 75-100ms (H) and 120-180ms (I) epochs. 
bioRxiv preprint doi: https://doi.org/10.1101/2022 02 11.480080; this version posted February 14,2022 . The copyright holder for this preprint (which was not certified by peer review) is the author/funder, who has granted bioRxiv a license to display the preprint in perpetuity. It is made available under aCC-BY-NC-ND 4.0 International license.

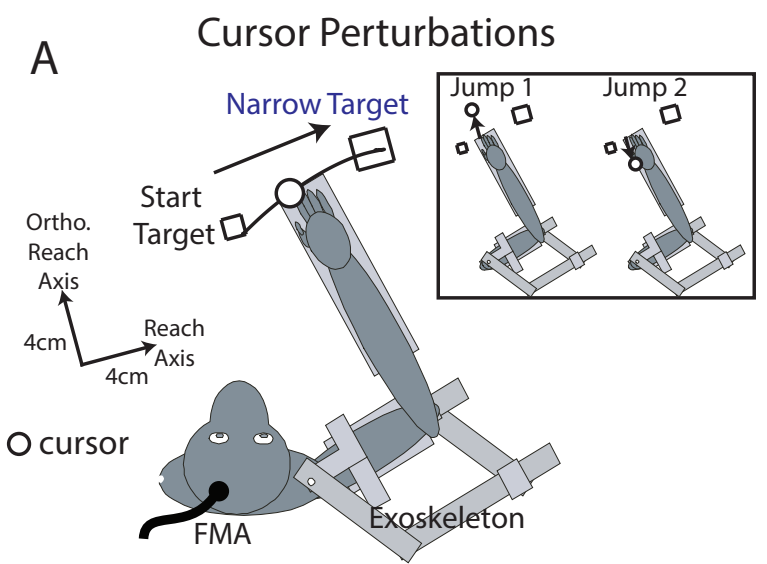

Wide Target

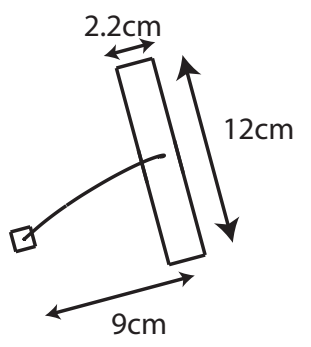

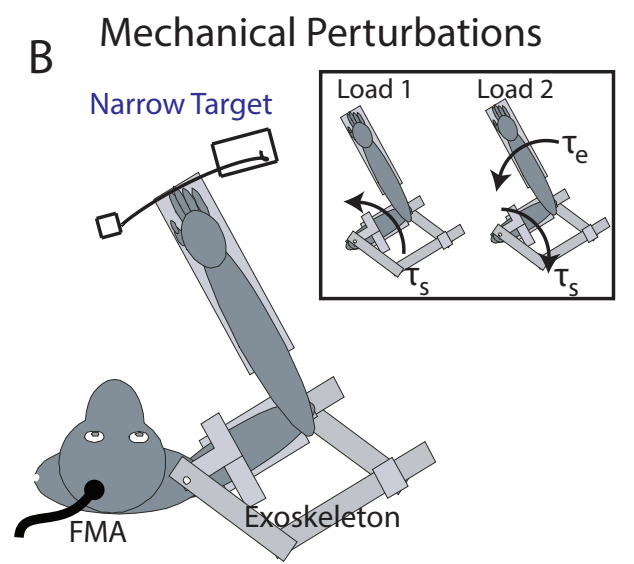

Wide Target

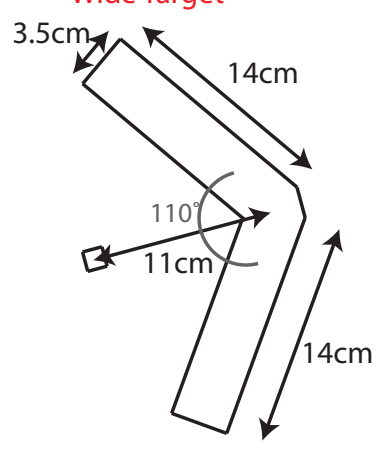

Figure 1 
A
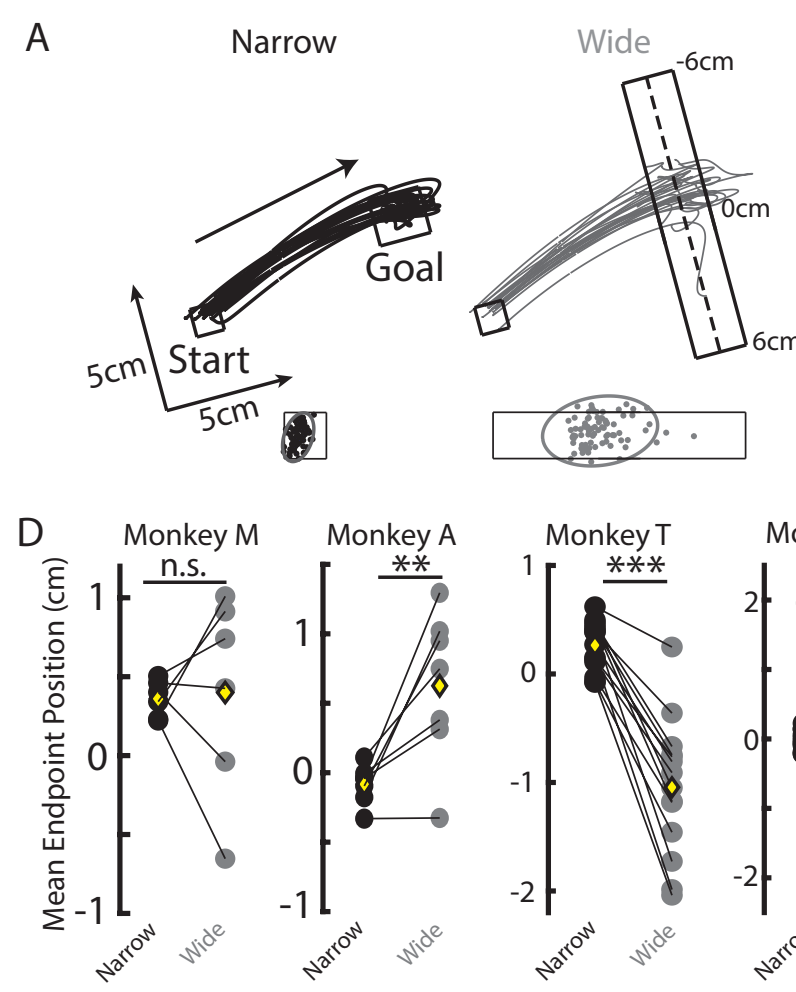
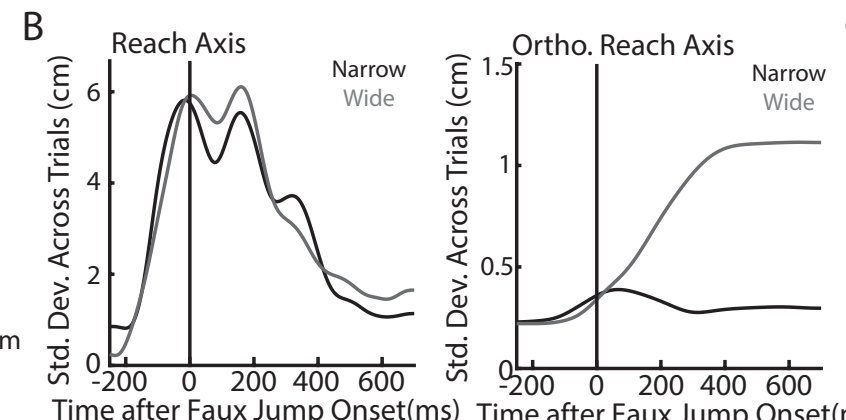

C

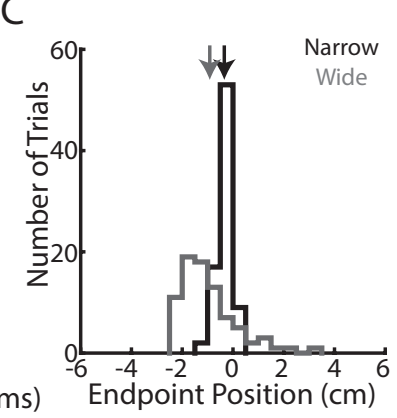

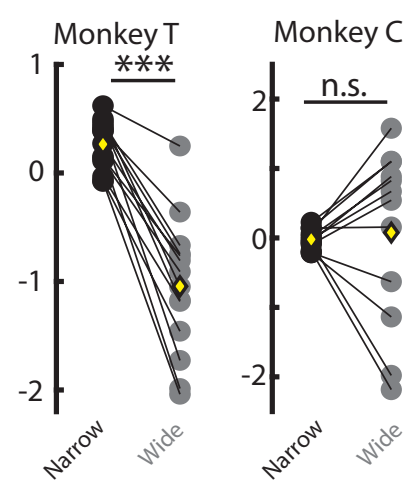
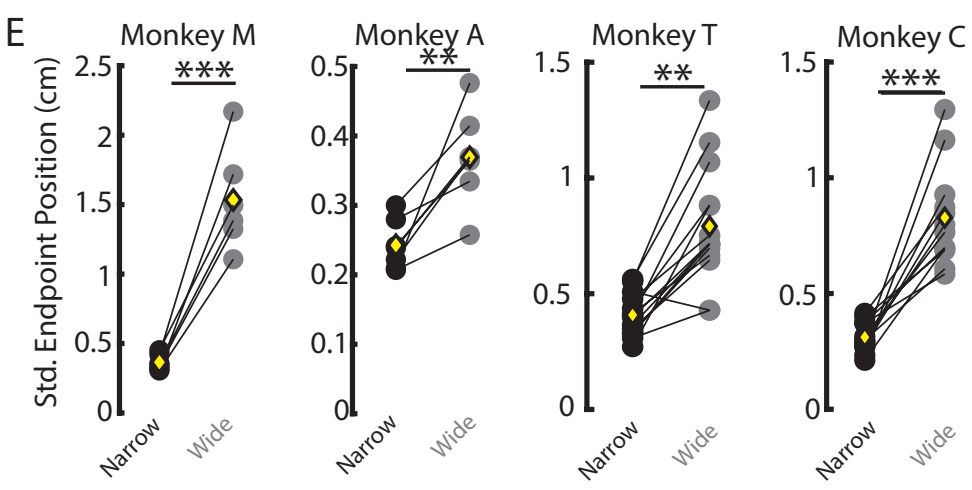

Figure 2 
bioRxiv preprint dol: https://doi.org/10.1101/2022.02.11.480080; this version posted February 14, 2022. The copyright holder for this preprint (which was not certified by peer review) is the author/funder, who has granted bioRxiv a license to display the preprint in perpetuity. It is made available under aCC-BY-NC-ND 4.0 International license.
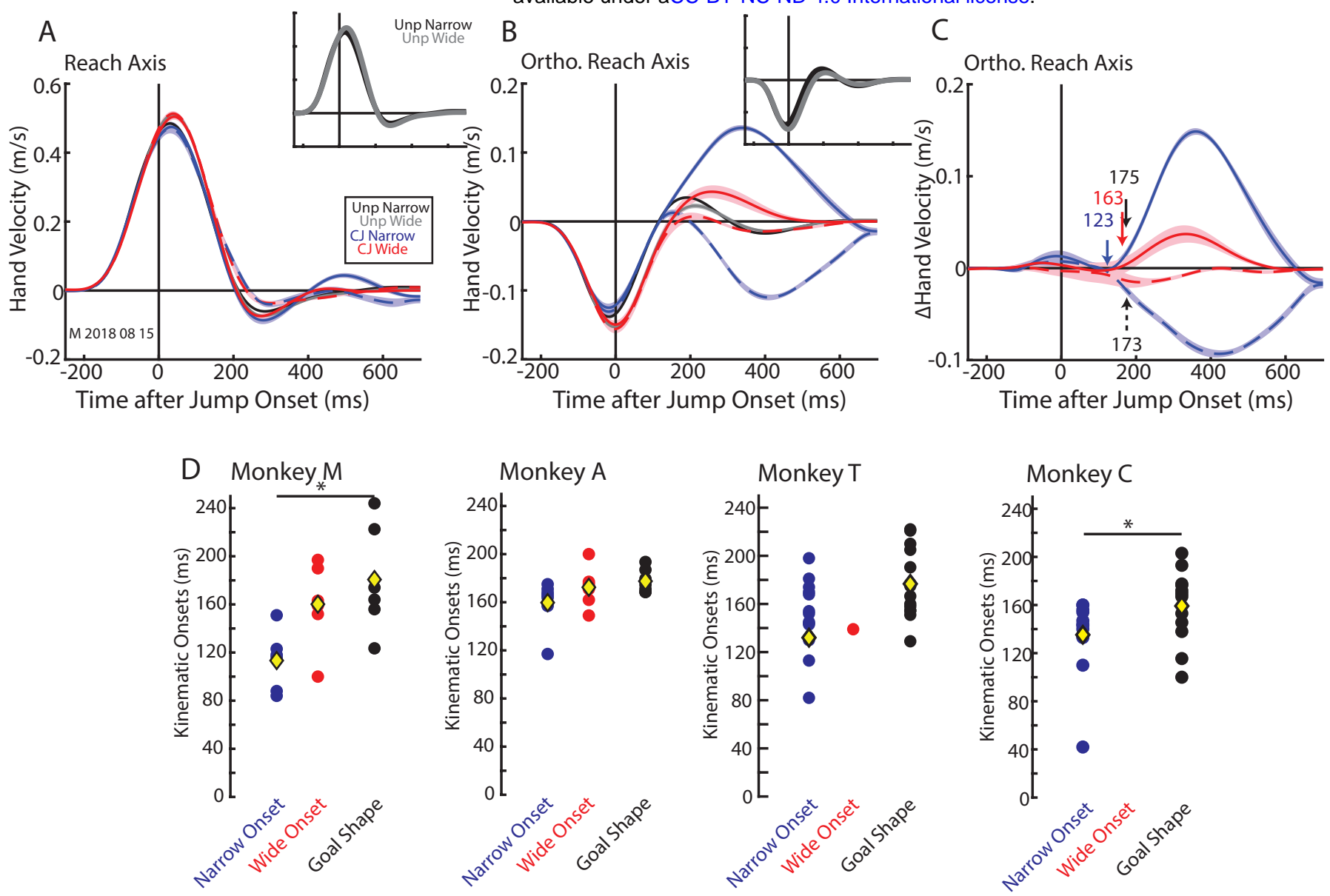

Figure 3 
A

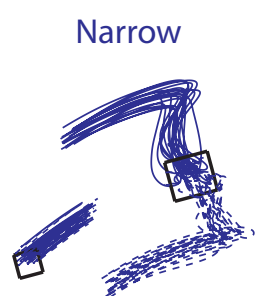

- Jump Away from Body

- - - Jump Towards Body Jump Away from Body

Jump Towards Body

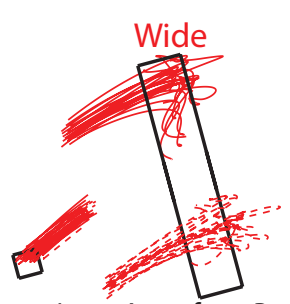

- Jump Away from Body
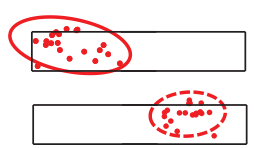

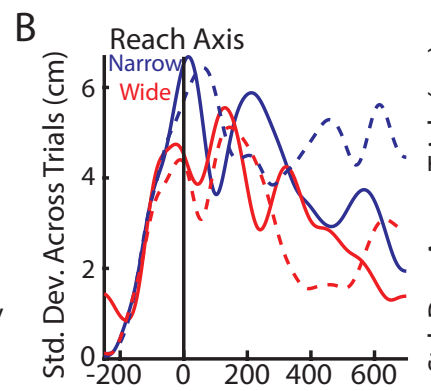

Time after Jump Onset(ms)

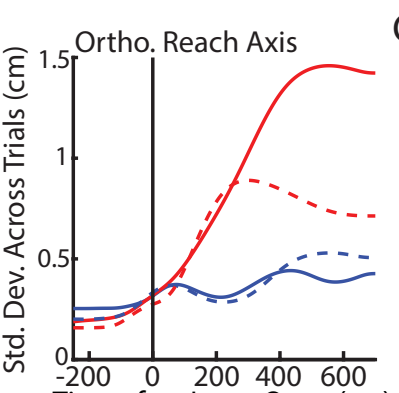

Time after Jump Onset(ms)

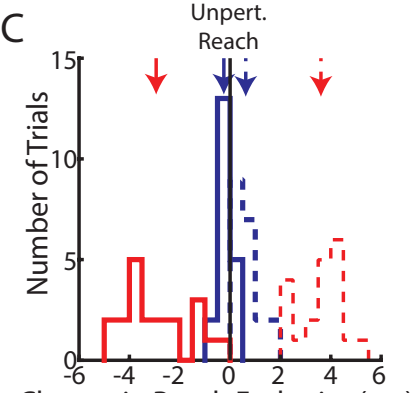

Change in Reach Endpoint (cm)
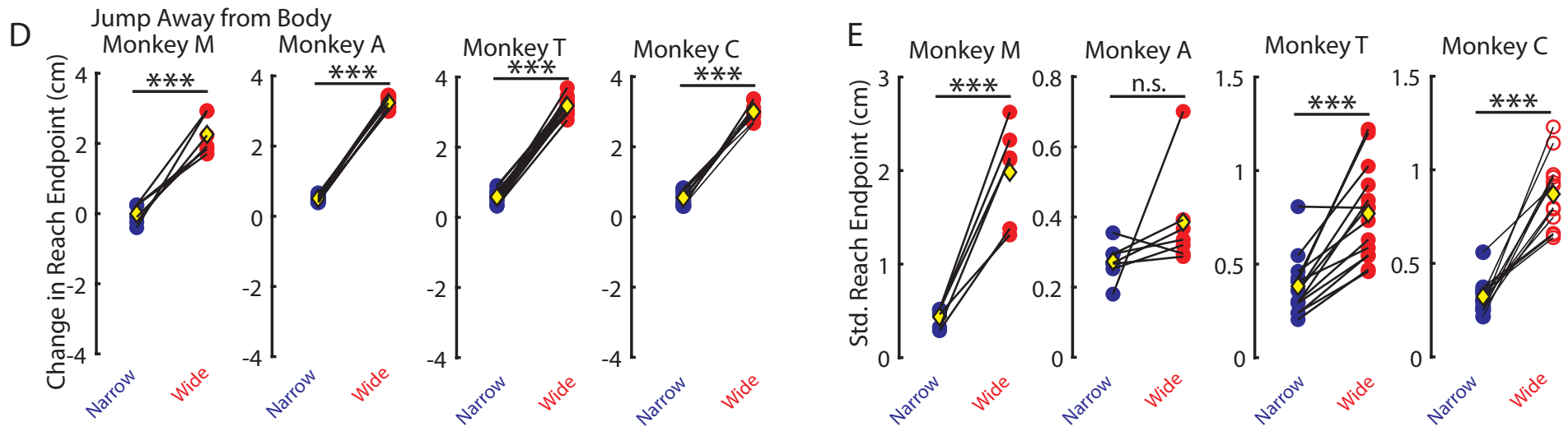

F Jump Towards Body
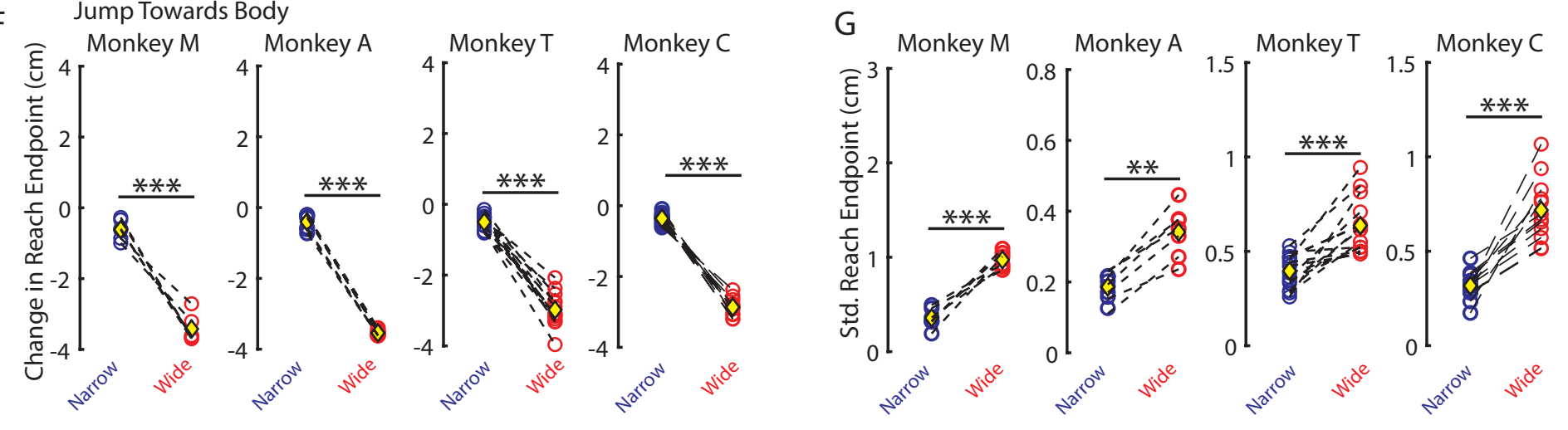

Figure 4 
A

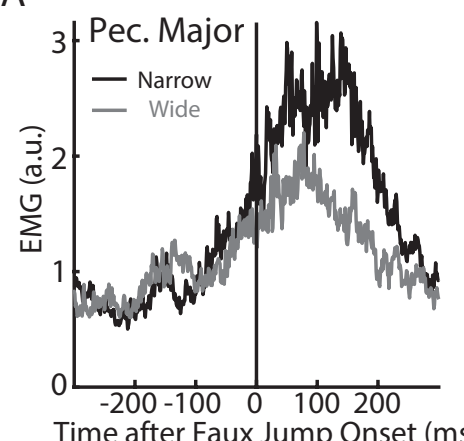

B

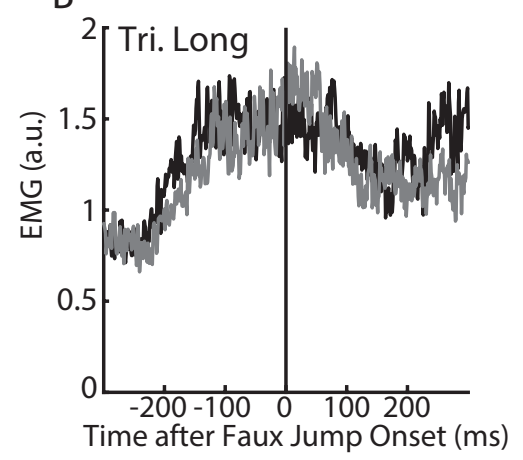

C

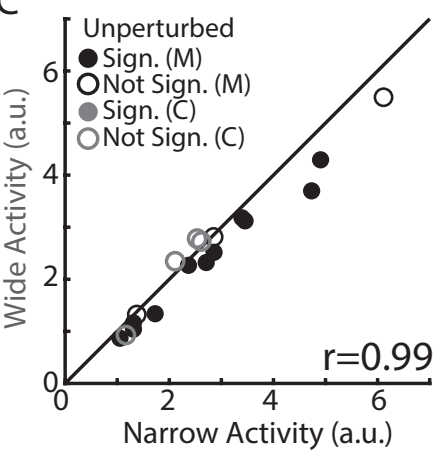

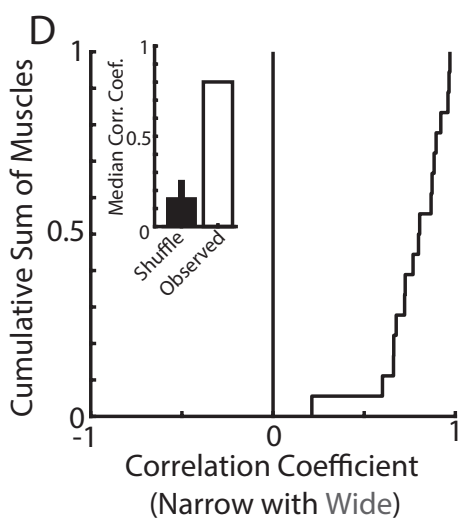

Figure 5 


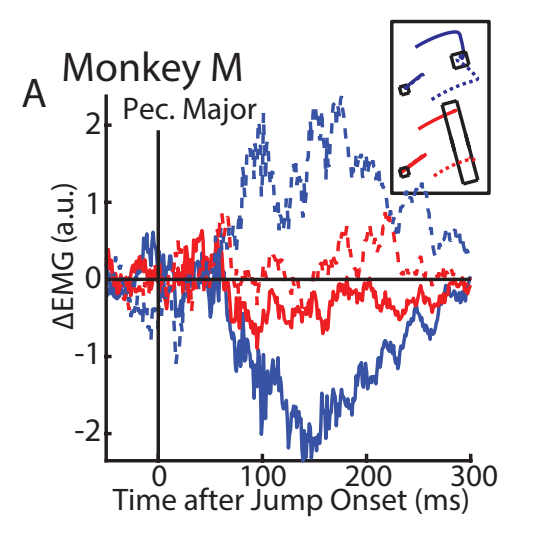

F

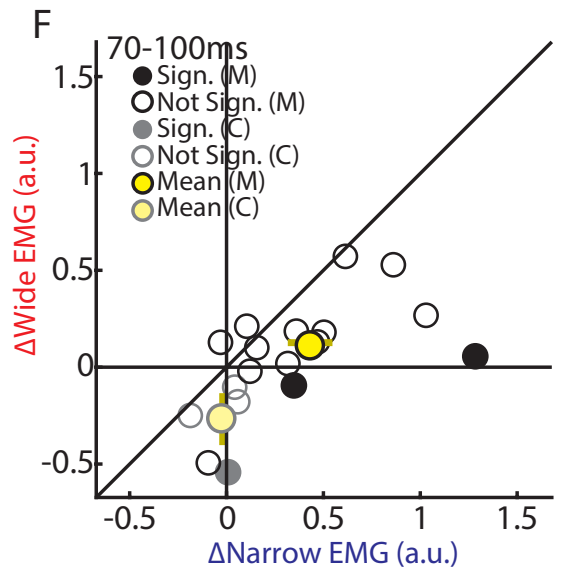

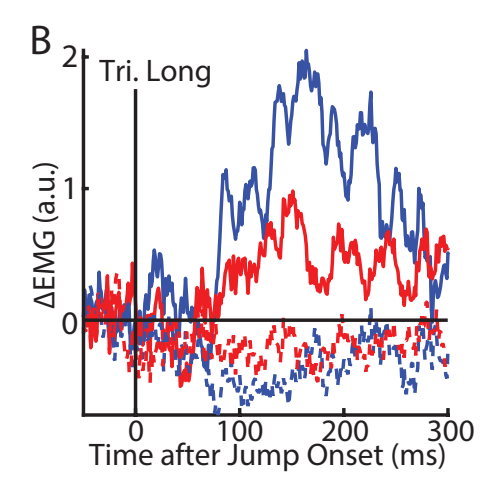

G

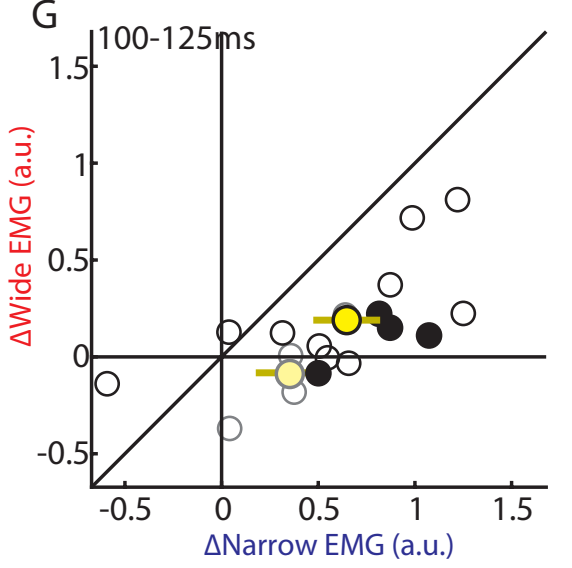

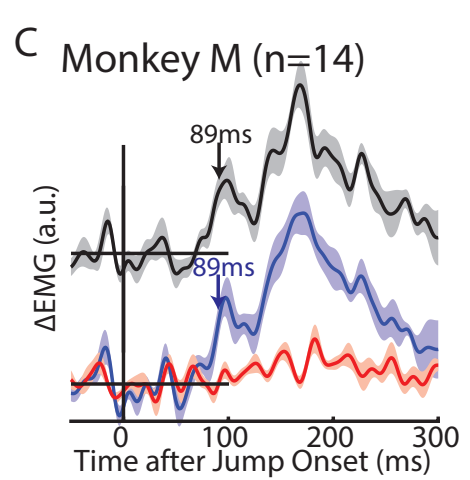

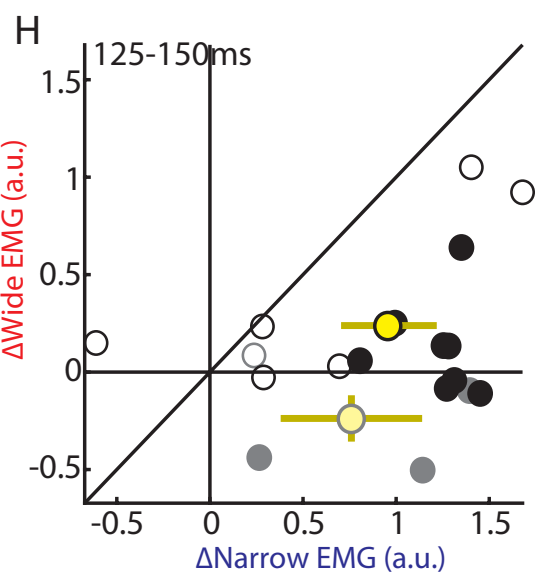

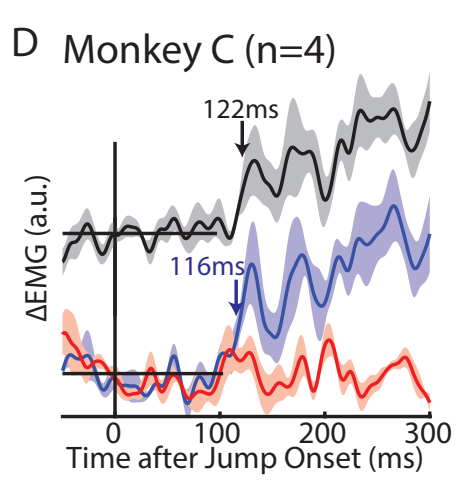
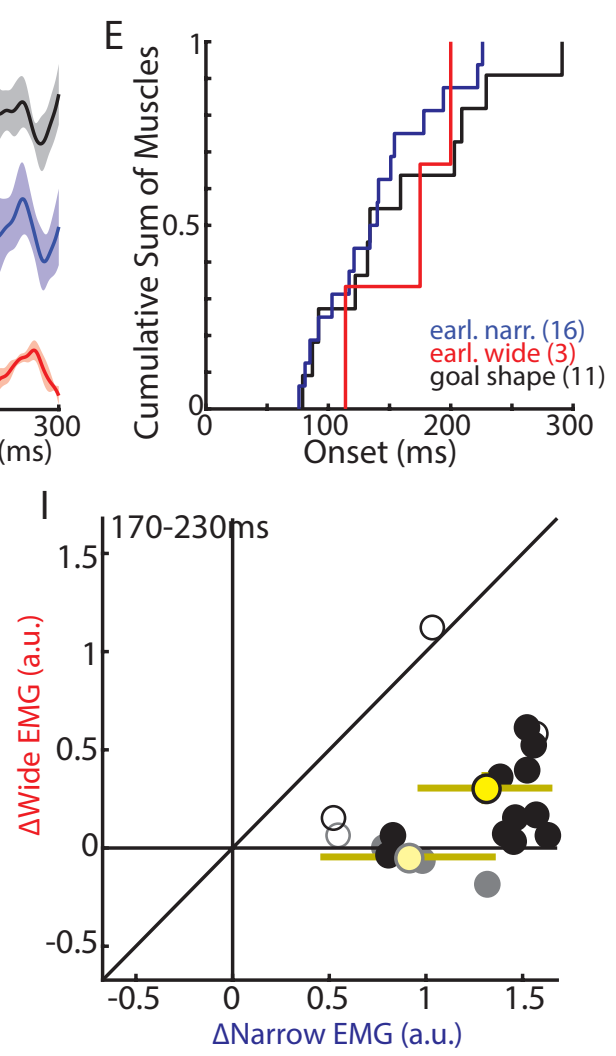

Figure 6 
bioRxiv preprint dol: https://doi.org/10.1101/2022.02.11.480080; this version posted February 14, 2022. The copyright holder for this preprint (which was not certified by peer review) is the author/funder, who has granted bioRxiv a license to display the preprint in perpetuity. It is made

A
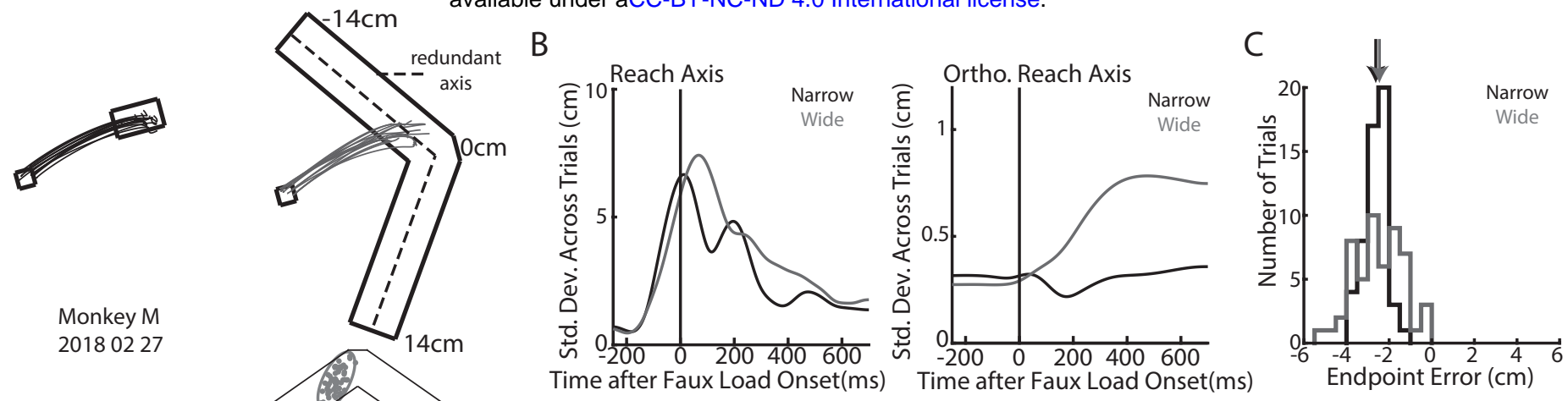

D
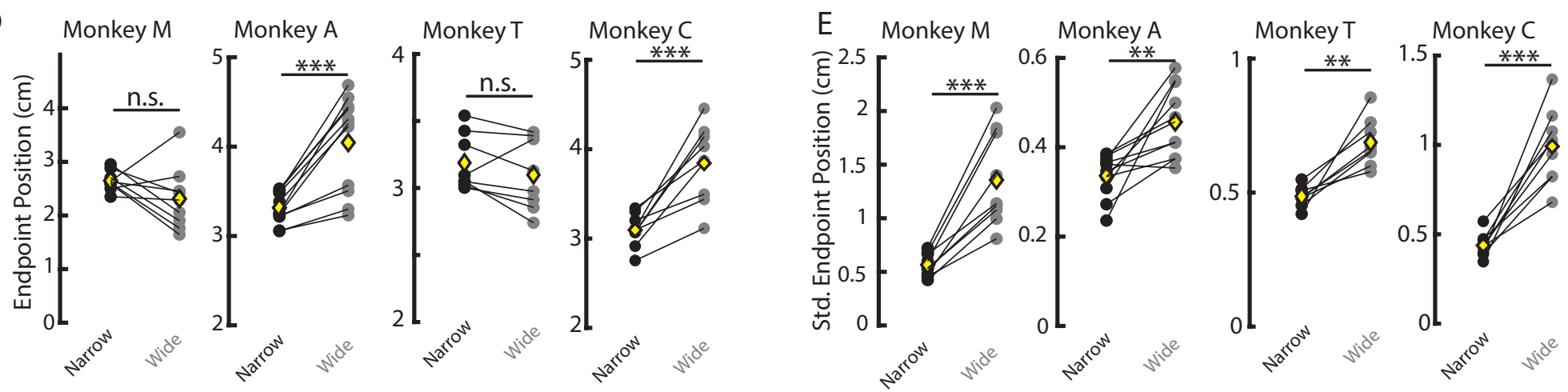

Figure 7 

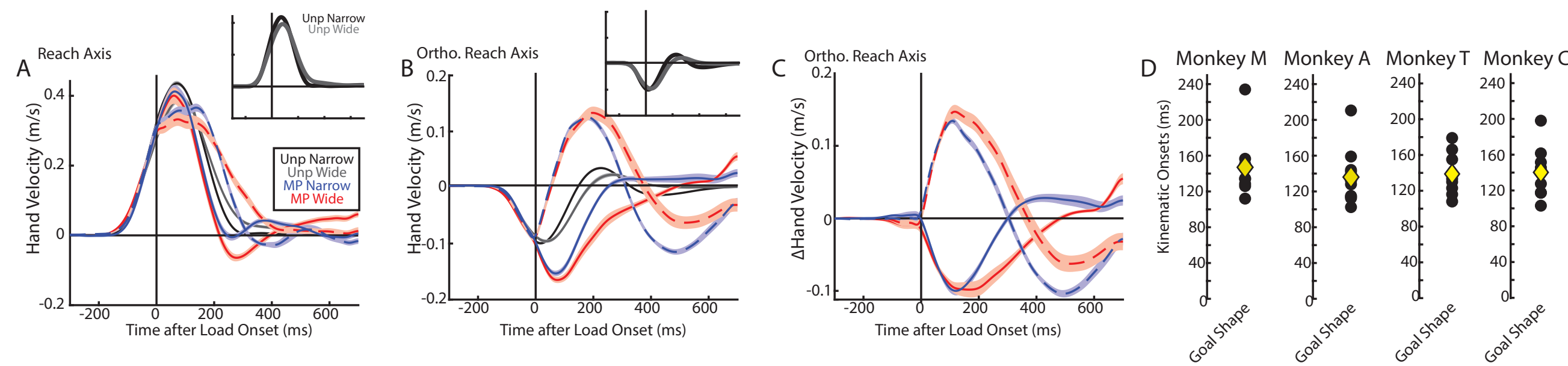

Figure 8 
A

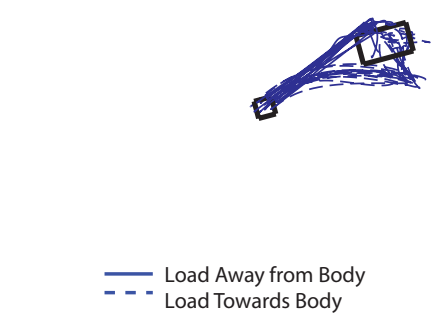

Load Away from Body

(6)

Load Towards Body

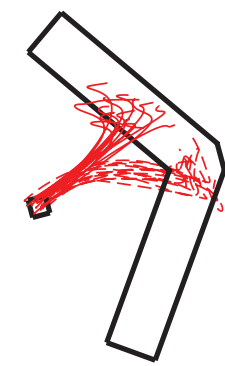

- - Load Away from Body
- Load Towards Body

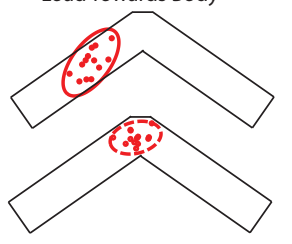

$\mathrm{B}$

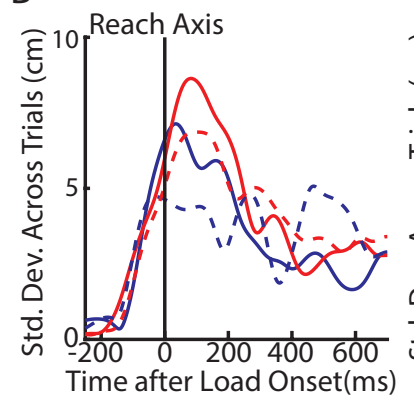

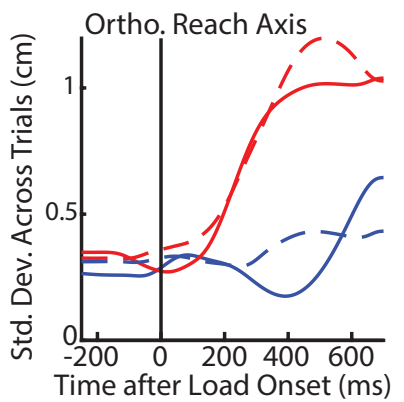

C

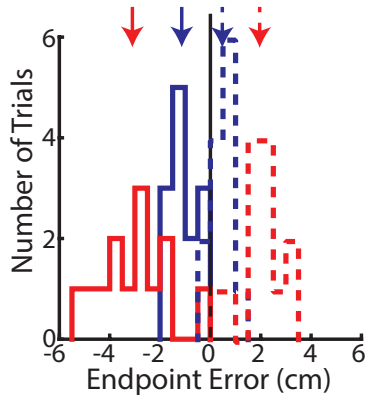

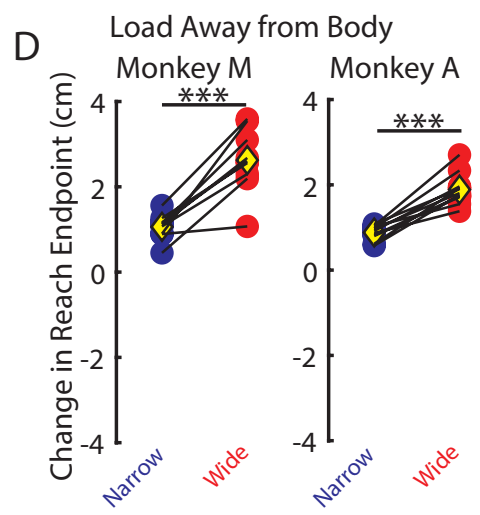
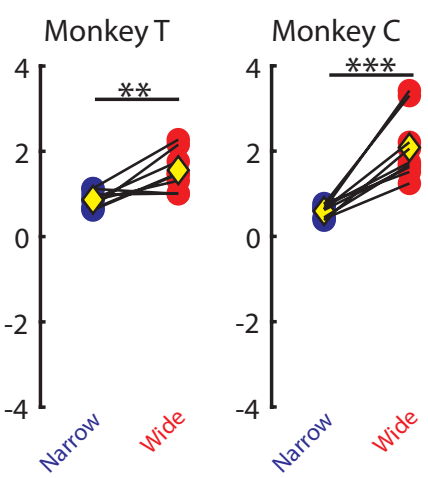

$F$ Monkey M

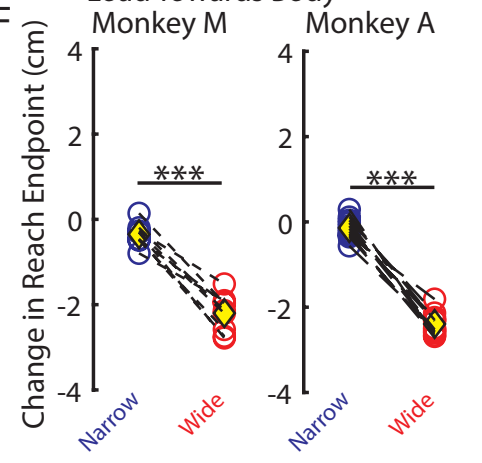

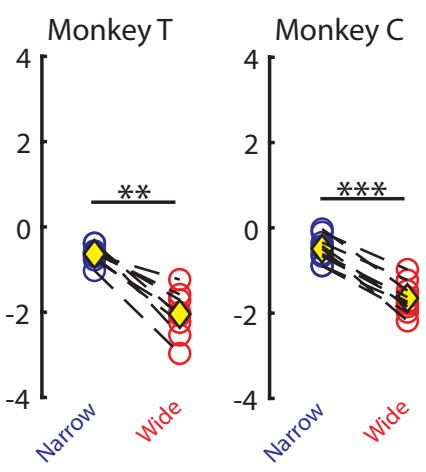

E
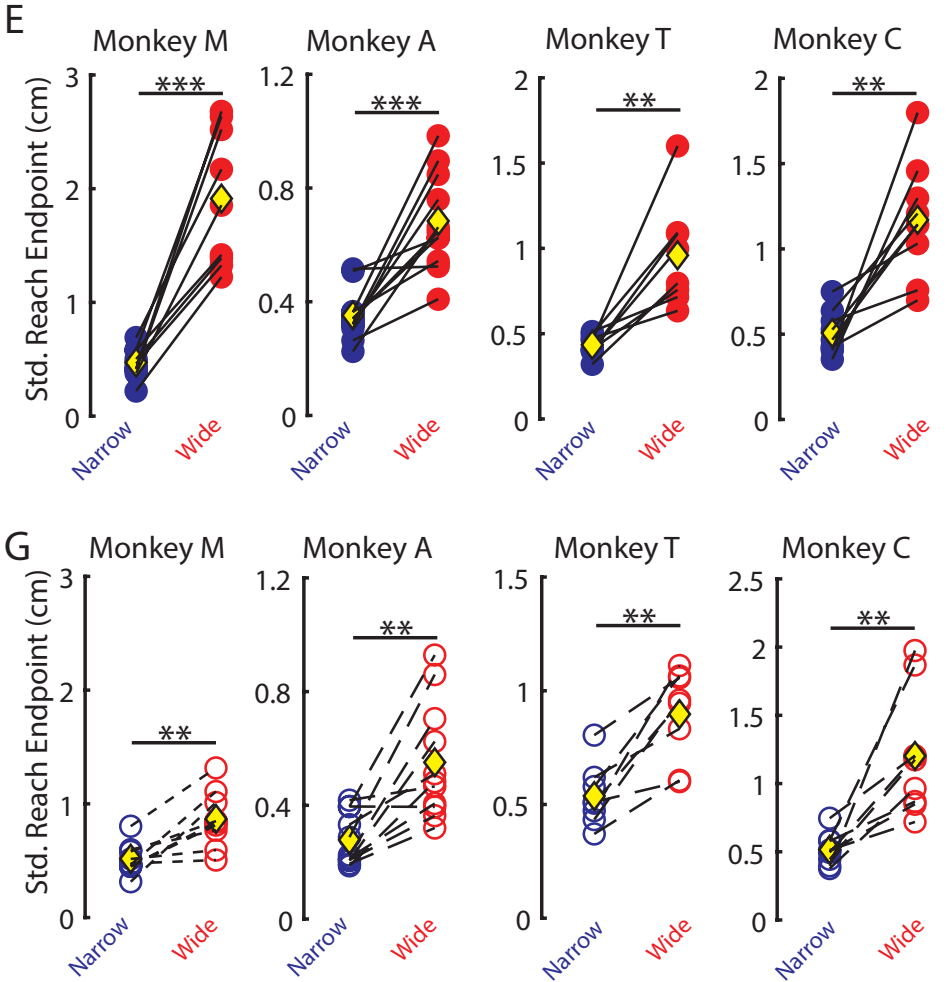

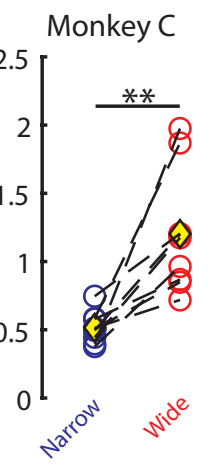

Figure 9 


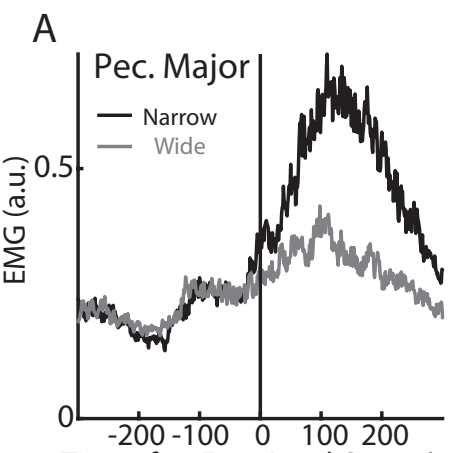

Time after Faux Load Onset (ms)
B

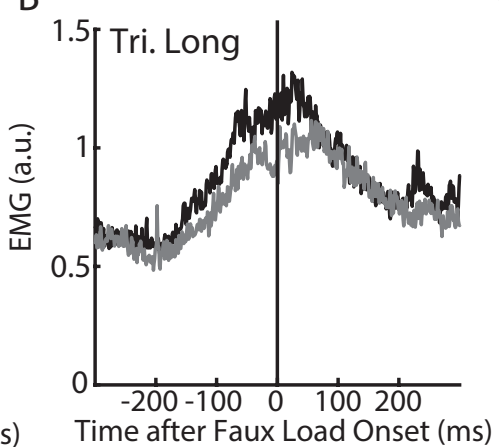

C

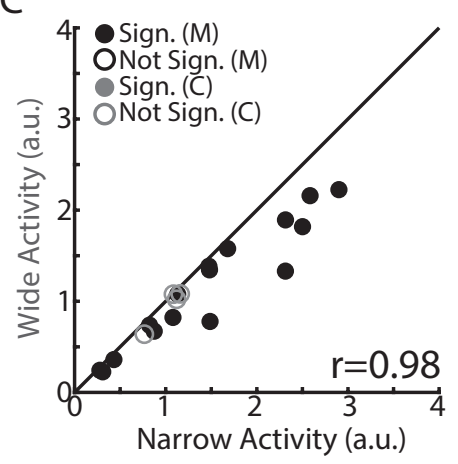

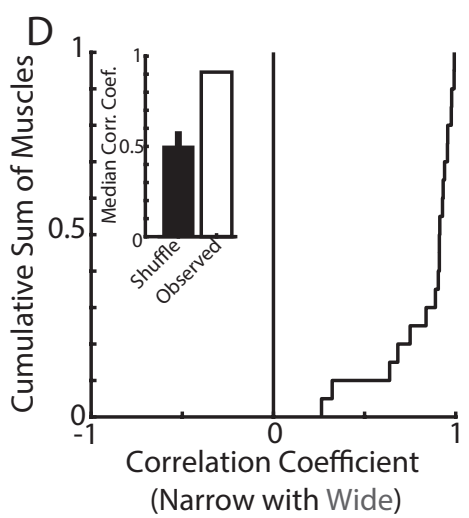

Figure 10 

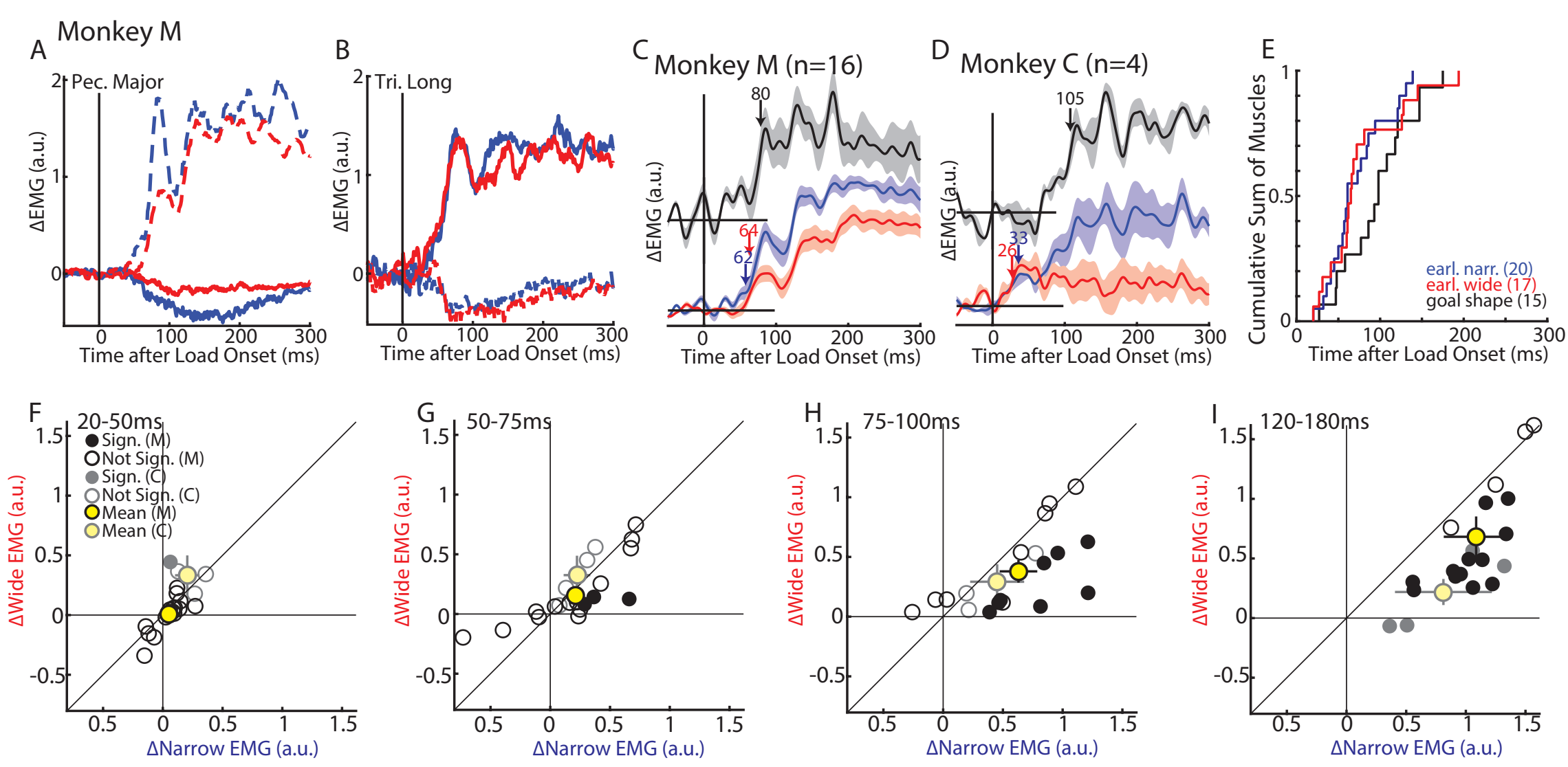

Figure 11 